\title{
CONSERVATION LAWS OF INVISCID NON-ISENTROPIC COMPRESSIBLE FLUID FLOW IN $n>1$ SPATIAL DIMENSIONS
}

\author{
STEPHEN C. ANCO ${ }^{1}$ AND AMANULLAH DAR ${ }^{1,2}$ \\ ${ }^{1}$ DePARTMENT OF MATHEMATiCs, BROCK UNIVERsity, ST. CATHARINES, ON CANADA \\ ${ }^{2}$ DEPARTMENT OF MATHEMATICS, QUAID-E-AZAM UNIVERSiTy, ISLAMABAD, PAKISTAN
}

\begin{abstract}
Recent work giving a classification of kinematic and vorticity conservation laws of compressible fluid flow with barotropic equations of state (where pressure is a function only of the fluid density) in $n>1$ spatial dimensions is extended to general non-isentropic equations of state in which the pressure is also a function of the dynamical entropy (per unit mass) of the fluid. Two main results are obtained. First, we find that apart from the familiar conserved integrals for mass, momentum, energy, angular momentum and Galilean momentum, and volumetric entropy, additional kinematic conserved integrals arise only for non-isentropic equations of state given by a generalized form of the well-known polytropic equation of state with dimension-dependent exponent $\gamma=1+2 / n$, such that the proportionality coefficient is an arbitrary function of the entropy (per unit mass). Second, we show that the only vorticity conserved integrals consist of a circulatory entropy (which vanishes precisely when the fluid flow is irrotational) in all even dimensions. In particular, the vorticity integrals for helicity in odd dimensions and enstrophy in even dimensions are found to be no longer conserved for any non-isentropic equation of state.
\end{abstract}

\section{INTRODUCTION AND SUMMARY}

The mathematical study of $n$-dimensional fluid flow has attracted rising interest in the past few decades, encompassing work on symmetries and conservation laws [21, 13, 14], Hamiltonian structures [25], Casimir invariants [22, 12, 15], and other group-theoretic aspects of the $n$-dimensional Eulerian fluid equations [7, 8, 9]. Further results in the special case of $n=1$ dimension appear in [23, 24, 20].

In a recent contribution [5], we undertook a systematic study of local conservation laws for the Euler equations governing isentropic compressible fluid flow in $n>1$ spatial dimensions, where the pressure of the fluid is a function of the fluid density as given by some barotropic equation of state, while the entropy (per unit mass) of the fluid is constant throughout the fluid domain. Our results fully settled the problem of finding all $n$-dimensional local continuity equations in two cases of primary interest: kinematic conservation laws, like mass, momentum and energy, for which the conserved density and spatial flux involve only the fluid velocity, density and pressure, in addition to the time and space coordinates; and

2000 Mathematics Subject Classification. Primary: 76N99, 37K05, 70S10; Secondary: 76M60.

Key words and phrases. compressible fluid, non-isentropic, conserved quantity, conservation law, continuity equation, helicity, enstrophy, circulation, Euler equations.

S.C.A. is supported by an NSERC research grant. A.D. thanks HEC, Pakistan, for providing a 6-month fellowship grant and the Department of Mathematics at Brock University for additional support during the extended period of a visit when this research was completed. 
vorticity conservation laws, such as helicity in three dimensions as well as circulation and enstrophy in two dimensions, where the conserved density and spatial flux have an essential dependence on the curl of the fluid velocity. These two classes of conservation laws comprise all of the local continuity equations known to-date for isentropic compressible fluid flow in $n>1$ dimensions.

The present paper extends this work to non-isentropic adiabatic compressible fluid flow in which the entropy (per unit mass) is conserved only along streamlines and the pressure is given by an equation of state in terms of both the fluid density and entropy. In particular, we explicitly derive all $n$-dimensional kinematic and vorticity conservation laws, including any that are admitted only for special non-isentropic equations of state or in special dimensions $n>1$, with the conserved densities and spatial fluxes allowed to depend on the entropy (in addition to the previous fluid variables).

In section 2, we begin by reviewing the formulation of necessary and sufficient equations for directly determining the conserved densities admitted by the Euler equations for $n$-dimensional inviscid non-isentropic compressible fluid flow. We next verify that the physically familiar kinematic conserved integrals for mass, momentum, energy, angular momentum and Galilean momentum in compressible fluid flow with a general isentropic equation of state remain conserved for the $n$-dimensional non-isentropic Euler equations. By solving the determining equations for kinematic conserved densities in $n>1$ dimensions, we then show that the only additional conserved integrals consist of volumetric entropy in a generalized form, plus two generalized energies arising for polytropic equations of state where the pressure is proportional to a particular dimension-dependent power $\gamma=1+2 / n$ of the density, with the proportionality coefficient given by an arbitrary function of the entropy (per unit mass).

In section 3, we first consider the odd-dimensional helicity integral and the evendimensional enstrophy integral both of which are known to be conserved in $n$-dimensional isentropic fluid flow [15, 5]. By examining how the corresponding local vorticity conservation laws depend on the equation of state of the fluid, we show that the helicity and enstrophy integrals are no longer conserved for any non-isentropic equations of state. Next we solve the determining equations to see if there are any vorticity conserved densities in $n>1$ dimensions admitted by the non-isentropic Euler equations. As a main new result, this classification yields a non-trivial conserved integral given by an underlying local conservation law describing a circulatory entropy (which vanishes whenever the fluid is irrotational) in all even dimensions.

Next, in section 4, we classify kinematic and vorticity constants of motion on $n$-dimensional domains that move with the fluid. Such constants of motion arise from non-trivial local conservation laws such that the net flux through the moving boundary of the domain is zero. The main result of this classification is to show that, firstly, there are no vorticity constants of motion and, secondly, the only kinematic constants of motion are given by the mass integral and the volumetric entropy integral defined on any moving domain in the fluid. We also investigate possibilities for constants of motion defined by a conserved integral on the boundary of a moving domain, in analogy with Kelvin's circulation integral in the case of isentropic fluid flow in $n=2$ dimensions. Such constants of motion correspond to conservation laws satisfying the condition of zero net flux on the moving boundary but such that the conserved density in the moving domain is locally trivial (i.e. having the form of a spatial divergence). From our classification proofs for kinematic and vorticity conservation 
laws, we find that there are no moving-boundary constants of motion for non-isentropic compressible fluid flow in $n>1$ dimensions, in contrast to the generalized circulation integral which exists in the isentropic case for all even dimensions as found in [5].

In section 5, we then use a Hamiltonian formulation of the non-isentropic compressible Euler equations to classify all Hamiltonian symmetries corresponding to the kinematic and vorticity conservation laws in $n>1$ dimensions.

In section 6, based on the well-known relationship between the non-isentropic compressible Euler equations and the equations of gas dynamics, we state a complete classification of conservation laws of kinematic and vorticity forms for gas dynamics in $n>1$ dimensions. In this classification, the fluid equation of state gets replaced by a state function that specifies both the speed of sound in the gas and the thermodynamic energy density of the gas in terms of the gas pressure and density. We thereby obtain conserved integrals for mass, momentum, energy, angular momentum and Galilean momentum, plus volumetric entropy and, in even dimensions, circulatory entropy, holding for all state functions. The only distinguished state function for which the gas dynamics equations admit additional conserved integrals is shown to be the polytropic case, where the state function is proportional to the pressure, with a particular dimension-dependent proportionality coefficient $\gamma=1+2 / n$.

Finally, in section 7, we summarize our classification of fluid flow conservation laws in index notation and also list the multipliers that correspond to each non-trivial conserved density. Proofs of the main classification theorems are given in two appendices.

An interesting question left open for future work is to extend these classifications to conservation laws that depend on derivatives of the curl of the velocity or more generally on higher derivatives of the velocity itself.

\section{Non-ISENTROPic COMPRESSible Euler Equations}

Compressible non-isentropic fluids in $\mathbb{R}^{n}$ are described (in the absence of external forces and viscosity) by a generalization of the Euler equations such that the equation of state for pressure becomes a function of the entropy in addition to the density:

$$
\begin{aligned}
& p=P(\rho, S), \\
& \partial_{t} \mathbf{u}+\mathbf{u} \cdot \nabla \mathbf{u}+\frac{1}{\rho} \nabla p=0, \\
& \partial_{t} \rho+\nabla \cdot(\rho \mathbf{u})=0 .
\end{aligned}
$$

Here $\mathbf{u}(t, \mathbf{x})$ is velocity; $\rho(t, \mathbf{x})$ is density; and $S(t, \mathbf{x})$ is entropy (per unit mass) of the fluid which is conserved along streamlines

$$
\partial_{t} S+\mathbf{u} \cdot \nabla S=0
$$

In particular when $S=$ const. or $\partial P / \partial S=0$, the system (2.1)-(2.3) reduces to the case of isentropic fluid flow studied in [5], where

$$
p=P(\rho)
$$

thus the non-isentropic case is characterized by having $\partial P / \partial S \not \equiv 0$ with $S$ being a dynamical variable which is non-constant across streamlines. 
We will study local conservation laws of the Eulerian system (2.1)-(2.4). Similar to the isentropic case, conservation laws of non-isentropic fluid flow are described by a local continuity equation

$$
D_{t} T+D_{\mathbf{x}} \cdot \mathbf{X}=0
$$

holding formally for all solutions of (2.1) -(2.4), where the conserved density $T$ and spatial flux $\mathbf{X}$ are some functions of $t, \mathbf{x}, \mathbf{u}, \rho, S$, and $\mathbf{x}$-derivatives of $\mathbf{u}, \rho, S$. Here $D_{t}$ and $D_{\mathbf{x}}$ denote total time and space derivatives respectively. In integral form, the continuity equation (2.6) is equivalently given by

$$
\frac{d}{d t} \int_{V} T d^{n} x=-\int_{\partial V} \mathbf{X} \cdot \hat{\mathbf{n}} d^{n-1} \sigma
$$

where $V$ is any spatial domain in $\mathbb{R}^{n}$ through which the fluid is flowing, $\hat{\mathbf{n}}$ is the outward unit normal and $d^{n-1} \sigma$ denotes the surface element of the domain boundary $\partial V$. Alternatively, conservation laws (2.6) and (2.7) can be formulated by considering a spatial domain $V(t)$ that moves with the fluid. Then the spatial flux through the moving boundary $\partial V(t)$ is $\boldsymbol{\xi}=\mathbf{X}-T \mathbf{u}$ which is related to the conserved density $T$ by the transport equation

$$
D_{t} T+\mathbf{u} \cdot D_{\mathbf{x}} T=-(\nabla \cdot \mathbf{u}) T-D_{\mathbf{x}} \cdot \boldsymbol{\xi}
$$

where $D_{t}+\mathbf{u} \cdot D_{\mathbf{x}}$ represents the total convective (material) derivative and $\nabla \cdot \mathbf{u}$ represents the expansion or contraction of an infinitesimal volume moving with the fluid. The corresponding integral form of a fluid conservation law in a moving domain is thereby expressed as

$$
\frac{d}{d t} \int_{V(t)} T d^{n} x=-\int_{\partial V(t)} \boldsymbol{\xi} \cdot \hat{\mathbf{n}} d^{n-1} \sigma
$$

in terms of the moving-flux $\boldsymbol{\xi}$ through the domain boundary $\partial V(t)$.

For determining conserved densities $T$, we note the local continuity equation (2.6) shows that $\mathcal{D}_{t} T$ must have the form of a spatial divergence $D_{\mathbf{x}} \cdot(-\mathbf{X})$, where $\mathcal{D}_{t}$ is the total time derivative evaluated on solutions of (2.1)-(2.4). Hence, necessary and sufficient equations [10] for finding conserved densities $T$ are given by

$$
E_{\mathbf{u}}\left(\mathcal{D}_{t} T\right)=E_{\rho}\left(\mathcal{D}_{t} T\right)=E_{S}\left(\mathcal{D}_{t} T\right)=0
$$

where $E_{\mathbf{u}}, E_{\rho}, E_{S}$ are spatial Euler operators with respect to $\mathbf{u}, \rho, S$. To-date, a complete classification of all conserved densities $T(t, \mathbf{x}, \mathbf{u}, \rho, S, \nabla \mathbf{u}, \nabla \rho, \nabla S, \ldots)$ has not appeared in the literature.

Throughout, on $\mathbb{R}^{n}$, $\nabla$ will denote the gradient operator, while $\cdot$ and $\wedge$ will respectively denote the Euclidean inner product and exterior (antisymmetric) product.

2.1. Classification of kinematic conservation laws. For $n$-dimensional compressible fluid flow, we first write down the well-known kinematic conservation laws [14, 5] for mass, momentum, angular momentum, Galilean momentum, and energy in terms of the fluid velocity $\mathbf{u}$, density $\rho$ and pressure $p$, on any spatial domain $V(t) \subset \mathbb{R}^{n}$ transported in the 
fluid:

$$
\begin{aligned}
& \frac{d}{d t} \int_{V(t)} \rho d^{n} x=0, \\
& \frac{d}{d t} \int_{V(t)} \rho \mathbf{u} d^{n} x=-\int_{\partial V(t)} p \hat{\mathbf{n}} d^{n-1} \sigma, \\
& \frac{d}{d t} \int_{V(t)} \rho \mathbf{u} \wedge \mathbf{x} d^{n} x=\int_{\partial V(t)} p \mathbf{x} \wedge \hat{\mathbf{n}} d^{n-1} \sigma, \\
& \frac{d}{d t} \int_{V(t)} \rho(t \mathbf{u}-\mathbf{x}) d^{n} x=-\int_{\partial V(t)} t p \hat{\mathbf{n}} d^{n-1} \sigma, \\
& \frac{d}{d t} \int_{V(t)} \rho\left(\frac{1}{2}|\mathbf{u}|^{2}+e\right) d^{n} x=-\int_{\partial V(t)} p \mathbf{u} \cdot \hat{\mathbf{n}} d^{n-1} \sigma .
\end{aligned}
$$

Here $e$ is the internal (thermodynamic) energy density defined in terms of $p$ and $\rho$ by

$$
\left.\frac{\partial e}{\partial \rho}\right|_{S=\text { const. }}=\rho^{-2} p .
$$

We note that equation (2.16) is equivalent to the familiar thermodynamic relation [17, 26] $d(e+p / \rho)=(1 / \rho) d p$ holding for any adiabatic infinitesimal change $d S=0$ in the state of the fluid, where $e+p / \rho$ is the enthalpy.

Each of these conservation laws can be readily verified to hold using just the dynamical Euler equations (2.2) $-(2.3)$ for $\mathbf{u}$ and $\rho$, plus the transport equation (2.4) for $S$ in the case of the energy conservation law. As a consequence, the form of the conserved mass, momentum, angular momentum, and Galilean momentum is independent of the equation of state for $p$, and hence the conserved integrals (2.11), (2.12), (2.13), (2.14) are valid for non-isentropic fluid flow. In contrast, the form of the conserved energy (2.15) explicitly depends on the equation of state for $p$ in terms of both $\rho$ and $S$ through the relation (2.16) for the internal energy density. In particular, for fluid flow with a non-isentropic equation of state (2.1), the internal energy density is given by

$$
e(\rho, S)=\int \rho^{-2} P(\rho, S) d \rho
$$

Note this defines $e$ only up to an integration constant given by an arbitrary function of $S$. Such a function $f(S)$ contributes a term of the form $\rho f(S)$ to the energy density $E=$ $\rho\left(\frac{1}{2}|\mathbf{u}|^{2}+e\right)$ of the fluid, and as a result, conservation of energy yields the conservation law

$$
\frac{d}{d t} \int_{V(t)} \rho f(S) d^{n} x=0 .
$$

In the specific case $f(S)=S$, this conservation law (2.18) states that the volumetric entropy is conserved in any spatial domain transported by the fluid,

$$
\frac{d}{d t} \int_{V(t)} \rho S d^{n} x=0
$$

which is easy to check directly from the dynamical equations (2.3)-(2.4) for $\rho$ and $S$. The general case of the conservation law (2.18) can be understood to arise from conservation of 
volumetric entropy (2.19) by the freedom to functionally redefine the entropy $S$ to $\tilde{S}=f(S)$, preserving the form of the entropy transport equation (2.4).

The only other kinematic conservation laws known for compressible fluid flow in $n>1$ dimensions are the following generalized energies [14, 5]:

$$
\begin{aligned}
& \frac{d}{d t} \int_{V(t)}\left(t E-\frac{1}{2} \rho \mathbf{u} \cdot \mathbf{x}\right) d^{n} x=-\int_{\partial V(t)} p\left(t \mathbf{u}-\frac{1}{2} \mathbf{x}\right) \cdot \hat{\mathbf{n}} d^{n-1} \sigma \\
& \frac{d}{d t} \int_{V(t)}\left(t^{2} E-t \rho \mathbf{u} \cdot \mathbf{x}+\frac{1}{2} \rho|\mathbf{x}|^{2}\right) d^{n} x=-\int_{\partial V(t)} p t(t \mathbf{u}-\mathbf{x}) \cdot \hat{\mathbf{n}} d^{n-1} \sigma,
\end{aligned}
$$

each holding for a polytropic equation of state

$$
p=\kappa \rho^{1+2 / n}, \quad \kappa=\text { const. }
$$

where

$$
E=\rho\left(\frac{1}{2}|\mathbf{u}|^{2}+e\right)=\frac{1}{2} \rho|\mathbf{u}|^{2}+\frac{1}{2} n p
$$

is the polytropic energy density.

We now settle the natural questions of whether these generalized energies (2.20) $-(2.21)$ hold for any non-isentropic equations of state, and whether the non-isentropic compressible fluid equations (2.1)-(2.4) admit any additional conservation laws of kinematic form

$$
T(t, \mathbf{x}, \mathbf{u}, \rho, S) .
$$

Theorem 2.1. (i) For compressible fluid flow with a general non-isentropic equation of state (2.1), the admitted kinematic conservation laws 2.24) in any dimension $n>1$ comprise a linear combination of mass (2.11), momentum (2.12), angular momentum (2.13), Galilean momentum (2.14), energy (2.15)-(2.17), and generalized entropy (2.18). (ii) Modulo a constant shift in the pressure, the only equations of state for which additional conservation laws 2.24) arise for non-isentropic compressible fluid flow is the polytropic case

$$
p=\kappa(S) \rho^{1+2 / n}
$$

given in terms of an arbitrary function of the entropy, $\kappa(S)$. The admitted conservation laws consist of the similarity energy (2.20) and the Galilean energy (2.21) where $e(\rho, S)=$ $\frac{1}{2} n \kappa(S) \rho^{2 / n}$ is the internal energy density and $E=\rho\left(\frac{1}{2}|\mathbf{u}|^{2}+e\right)=\frac{1}{2} \rho|\mathbf{u}|^{2}+\frac{1}{2} n p$ is the polytropic energy density.

We give the proof of this classification theorem in Appendix A.

\section{VORTICITY CONSERVATION LAWS}

We will start by examining conservation of the odd-dimensional helicity integral and the even-dimensional enstrophy integral for compressible fluid flow in $n>1$ spatial dimensions. These integrals involve the spatial orientation tensor $\boldsymbol{\epsilon}$ contracted into products of the curl of the fluid velocity [5]

$$
\boldsymbol{\omega}=\nabla \wedge \mathbf{u}
$$

The dynamical equation for $\boldsymbol{\omega}$ is given by the curl of the Euler equation (2.2) for $\mathbf{u}$, combined with the identity $\mathbf{u} \cdot \boldsymbol{\omega}=\mathbf{u} \cdot \nabla \mathbf{u}-\frac{1}{2} \nabla(\mathbf{u} \cdot \mathbf{u})$, which yields

$$
\partial_{t} \boldsymbol{\omega}=\nabla \wedge(\boldsymbol{\omega} \cdot \mathbf{u})+\boldsymbol{\sigma}, \quad \nabla \wedge \boldsymbol{\omega}=0,
$$


where

$$
\boldsymbol{\sigma}=\nabla \rho^{-1} \wedge \nabla p, \quad \nabla \wedge \boldsymbol{\sigma}=0 .
$$

We note that in the case of an isentropic equation of state (2.5), this antisymmetric tensor (3.3) vanishes identically, since $\nabla p=P^{\prime}(\rho) \nabla \rho$ is proportional to $\nabla \rho$.

In odd dimensions $n=2 m+1 \geq 3$, the integral [9]

$$
\int_{V(t)} \mathbf{u} \cdot \varpi d^{n} x
$$

defines the fluid helicity on any spatial domain $V(t) \subset \mathbb{R}^{n}$ transported in the fluid, where

$$
\varpi=\boldsymbol{\epsilon} \cdot(\underbrace{(\nabla \wedge \mathbf{u}) \wedge \cdots \wedge(\nabla \wedge \mathbf{u})}_{(n-1) / 2 \text { times }})=*\left(\boldsymbol{\omega}^{m}\right)
$$

denotes the vorticity vector of the fluid. (Here $*$ is the Hodge dual operator applied to the rank $n-1=2 m$ skew-symmetric tensor $\boldsymbol{\omega}^{m}$.) Since $\boldsymbol{\omega}$ is curl-free, and the tensor $\boldsymbol{\epsilon}$ is constant and skew-symmetric, the vorticity vector (3.5) is divergence-free and obeys the transport equation

$$
\partial_{t} \varpi=-\nabla \cdot(\varpi \wedge \mathbf{u})+m \boldsymbol{\sigma} \cdot \mathbf{W}, \quad \mathbf{W}=*\left(\boldsymbol{\omega}^{m-1}\right),
$$

with

$$
\nabla \cdot \varpi=*\left(\nabla \wedge \boldsymbol{\omega}^{m}\right)=m(\nabla \wedge \boldsymbol{\omega}) \cdot *\left(\boldsymbol{\omega}^{m-1}\right)=0
$$

obtained from (3.2) and (3.5). Now for evaluating the time derivative of the helicity integral (3.4), we first use equations (3.6) and (2.2) to derive

$$
\partial_{t}(\mathbf{u} \cdot \varpi)=\nabla \cdot\left(-\mathbf{u}(\mathbf{u} \cdot \varpi)+\frac{1}{2} \varpi|\mathbf{u}|^{2}\right)-\rho^{-1} \varpi \cdot \nabla p-m \mathbf{W} \cdot(\mathbf{u} \wedge \boldsymbol{\sigma}) .
$$

We next rewrite the pressure gradient term in (3.8) by means of the identities $\mathbf{W} \cdot(\nabla \wedge \mathbf{u})=$ $\mathbf{W} \cdot \boldsymbol{\omega}=\varpi$ and $\nabla \cdot \mathbf{W}=(m-1)(\nabla \wedge \boldsymbol{\omega}) \cdot *\left(\boldsymbol{\omega}^{m-2}\right)=0$, yielding

$$
\rho^{-1} \varpi \cdot \nabla p=\nabla \cdot\left(\mathbf{W} \cdot(\mathbf{u} \wedge \nabla p) \rho^{-1}\right)+\mathbf{W} \cdot(\mathbf{u} \wedge \boldsymbol{\sigma})
$$

via integration by parts. We thus obtain

$$
\partial_{t}(\mathbf{u} \cdot \varpi)+\nabla \cdot\left(\mathbf{u}(\mathbf{u} \cdot \varpi)-\frac{1}{2} \varpi|\mathbf{u}|^{2}+\mathbf{W} \cdot(\mathbf{u} \wedge \nabla p) \rho^{-1}\right)=-(1+m) \mathbf{W} \cdot(\mathbf{u} \wedge \boldsymbol{\sigma})
$$

which has the form of a local continuity equation (2.6) up to the term proportional to $\boldsymbol{\sigma}$ on the right-hand side. This verifies conservation of helicity in the case of isentropic compressible fluid flow. For the general case of non-isentropic compressible fluid flow, helicity will be conserved if and only if, for a given equation of state, the non-vanishing $\boldsymbol{\sigma}$ term on the righthand side in equation (3.10) reduces to a total spatial divergence. Necessary and sufficient conditions for this to occur are that the spatial Euler operators $E_{\rho}, E_{S}, E_{\mathbf{u}}$ annihilate the $\boldsymbol{\sigma}$ term.

To proceed, for a general non-isentropic equation of state $p=P(\rho, S)$, we note $\nabla p=$ $P_{\rho} \nabla \rho+P_{S} \nabla S$ implies $\boldsymbol{\sigma}=-\rho^{-2} P_{S} \nabla \rho \wedge \nabla S=-\nabla e_{S} \wedge \nabla S$ with $e_{S} \not \equiv 0$, where $e$ is the internal (thermodynamic) energy density (2.17) defined in terms of $P(\rho, S)$. Hence, the antisymmetric tensor (3.3) in the non-isentropic case is given by the curl

$$
\boldsymbol{\sigma}=-\nabla \wedge\left(e_{S} \nabla S\right)
$$

This allows us to rewrite the right-hand side in equation (3.10)

$$
\left.\mathbf{W} \cdot(\mathbf{u} \wedge \boldsymbol{\sigma})=\nabla \cdot \underset{7}{(\mathbf{W}} \cdot \underset{7}{(\mathbf{u} \wedge} \nabla S) e_{S}\right)-e_{S} \nabla \cdot(\varpi S)
$$


via integration by parts. Similarly, we can rewrite the divergence term

$$
\nabla \cdot\left(\mathbf{W} \cdot(\mathbf{u} \wedge \nabla p) \rho^{-1}\right)=\nabla \cdot\left(\varpi\left(\rho^{-1} p+e\right)-e_{S} \mathbf{W} \cdot(\mathbf{u} \wedge \nabla S)\right)
$$

by means of the identity

$$
\nabla e=e_{\rho} \nabla \rho+e_{S} \nabla S=-p \nabla \rho^{-1}+e_{S} \nabla S=e_{S} \nabla S+\rho^{-1} \nabla p-\nabla\left(\rho^{-1} p\right)
$$

which follows from the thermodynamic relation (2.16). The conservation equation (3.10) thereby reduces to

$$
D_{t} T+D_{\mathbf{x}} \cdot(\mathbf{u} T)+D_{\mathbf{x}} \cdot \boldsymbol{\xi}=(1+m) e_{S} \nabla \cdot(\varpi S)
$$

with

$$
T=\mathbf{u} \cdot \varpi, \quad \boldsymbol{\xi}=\left(\rho^{-1} p+e-\frac{1}{2}|\mathbf{u}|^{2}\right) \varpi+m e_{S} \mathbf{W} \cdot(\nabla S \wedge \mathbf{u}) .
$$

We now observe the right-hand side in equation (3.15) fails to be a spatial divergence when the fluid flow is non-isentropic, since $P_{S} \not \equiv 0$ implies $E_{\rho}\left(e_{S} \nabla \cdot(\varpi S)\right)=e_{\rho S} \nabla \cdot(\varpi S)=$ $\rho^{-2} P_{S} \varpi \cdot \nabla S \not \equiv 0$. As a result, the helicity integral (3.4) obeys

$$
\begin{aligned}
\frac{d}{d t} \int_{V(t)} \mathbf{u} \cdot \varpi d^{n} x= & \int_{\partial V(t)}\left(\frac{1}{2}|\mathbf{u}|^{2}-\rho^{-1} p-e\right) \varpi \cdot \hat{\mathbf{n}} d^{n-1} \sigma+m \int_{\partial V(t)} e_{S} \mathbf{W} \cdot(\nabla S \wedge \mathbf{u} \wedge \hat{\mathbf{n}}) d^{n-1} \sigma \\
& +(1+m) \int_{V(t)} e_{S} \nabla \cdot(\varpi S) d^{n} x
\end{aligned}
$$

where the volume term depending on the function $e_{S}$ is not conserved for any non-isentropic equation of state (2.1).

In even dimensions $n=2 m \geq 2$,

$$
\int_{V(t)} \rho f(\varpi / \rho) d^{n} x
$$

defines the enstrophy integral [9] in terms of an arbitrary nonlinear function $f$ of $\varpi / \rho$, with

$$
\varpi=\boldsymbol{\epsilon} \cdot(\underbrace{(\nabla \wedge \mathbf{u}) \wedge \cdots \wedge(\nabla \wedge \mathbf{u})}_{n / 2 \text { times }})=*\left(\boldsymbol{\omega}^{m}\right)
$$

denoting the vorticity scalar of the fluid. (In the case when $f$ is a linear function of $\varpi / \rho$, the integral (3.18) reduces to the trivially conserved circulation integral, which will be discussed further in the next section.) The transport equation obeyed by $\varpi$ is given by

$$
\partial_{t} \varpi=-\nabla \cdot(\varpi \mathbf{u})-m \boldsymbol{\sigma} \cdot \mathbf{w}, \quad \mathbf{w}=*\left(\boldsymbol{\omega}^{m-1}\right)
$$

as obtained from (3.19) and (3.2). To now evaluate the time derivative of the enstrophy integral (3.18), we start by combining the transport equation (3.20) and the Euler equation (2.3) to get

$$
\partial_{t} \widetilde{\varpi}=-\mathbf{u} \cdot \nabla \widetilde{\varpi}-m \rho^{-1} \mathbf{w} \cdot \boldsymbol{\sigma}, \quad \widetilde{\varpi}=\varpi / \rho,
$$

and hence

$$
\partial_{t}(\rho f(\widetilde{\varpi}))+\nabla \cdot(\mathbf{u} \rho f(\widetilde{\varpi}))=m f^{\prime}(\widetilde{\varpi}) \mathbf{w} \cdot \boldsymbol{\sigma} .
$$

Thus, in the case of isentropic compressible fluid flow, conservation of enstrophy holds due to $\boldsymbol{\sigma}=0$. In the general case of non-isentropic compressible fluid flow, we can rewrite the right-hand side in equation (3.22) via

$$
\mathbf{w} \cdot \boldsymbol{\sigma}=-\nabla \cdot\left((\mathbf{w} \cdot \nabla S) e_{S}\right)
$$


which arises from the curl expression (3.11) for the antisymmetric tensor $\boldsymbol{\sigma}$ and from the divergence identity

$$
\nabla \cdot \mathbf{w}=*\left(\nabla \wedge \boldsymbol{\omega}^{m-1}\right)=(m-1)(\nabla \wedge \boldsymbol{\omega}) \cdot *\left(\boldsymbol{\omega}^{m-2}\right)=0 .
$$

We thereby obtain, after integration by parts,

$$
\mathcal{D}_{t} T+\nabla \cdot(\mathbf{u} T)+D_{\mathbf{x}} \cdot \boldsymbol{\xi}=-m e_{S} \nabla \cdot\left(\mathbf{w} \cdot \nabla S f^{\prime}(\widetilde{\varpi})\right)
$$

with

$$
T=\rho f(\widetilde{\varpi}), \quad \boldsymbol{\xi}=-m e_{S} f^{\prime}(\widetilde{\varpi}) \mathbf{w} \cdot \nabla S,
$$

where $f$ is a nonlinear function of $\widetilde{\varpi}$. Now, we find $E_{\rho}\left(e_{S} \nabla \cdot\left((\mathbf{w} \cdot \nabla S) f^{\prime}(\widetilde{\varpi})\right)\right)=$ $\rho^{-3} f^{\prime \prime}(\widetilde{\varpi}) P_{S} \mathbf{w} \cdot(\nabla \varpi \wedge \nabla S) \not \equiv 0$ due to $P_{S} \not \equiv 0$, and hence the right-hand side in equation (3.25) fails to be a spatial divergence since it is not annihilated by one of the spatial Euler operators. Consequently, for fluid flow with a non-isentropic equation of state, the enstrophy integral (3.18) on any spatial domain $V(t)$ transported in the fluid obeys

$$
\begin{aligned}
\frac{d}{d t} \int_{V(t)} \rho f(\varpi / \rho) d^{n} x= & -m \int_{\partial V(t)} e_{S} f^{\prime}(\varpi / \rho) \mathbf{w} \cdot(\nabla S \wedge \hat{\mathbf{n}}) d^{n-1} \sigma \\
& -m \int_{V(t)} e_{S} \nabla \cdot\left((\mathbf{w} \cdot \nabla S) f^{\prime}(\varpi / \rho)\right) d^{n} x
\end{aligned}
$$

where the volume term that depends on the function $e_{S}$ is not conserved.

Essentially, the underlying reason for non-conservation of both the enstrophy integral (3.18) and the helicity integral (3.4) for all non-isentropic equations of state is that $e_{S}=$ $\int \rho^{-2} P_{S} d \rho$ is necessarily a non-constant function of $\rho$.

We now address the open questions of whether any generalizations of the helicity (3.4) and enstrophy (3.18) are conserved for a non-isentropic equation of state or, more generally, whether the non-isentropic fluid equations (2.1)-(2.4) admit any conservation laws of vorticity form

$$
T\left(\rho, S, \mathbf{u}, *\left(\boldsymbol{\omega}^{m}\right)\right), \quad m \geq 1
$$

where

$$
*\left(\boldsymbol{\omega}^{m}\right)= \begin{cases}\varpi & \text { if } n=2 m \\ \varpi & \text { if } n=2 m+1\end{cases}
$$

is the vorticity scalar in even dimensions and the vorticity vector in odd dimensions, respectively.

Theorem 3.1. For any non-isentropic equation of state (2.1) the only non-trivial vorticity conservation laws (3.28) admitted for compressible fluid flow in dimensions $n>1$ are given by

$$
\frac{d}{d t} \int_{V(t)} f(S) \varpi d^{n} x=\int_{\partial V(t)} e_{S} \mathbf{w} \cdot(\nabla F(S) \wedge \hat{\mathbf{n}}) d^{n-1} \sigma
$$

for even dimensions $n=2 m$, where $f(S)=F^{\prime}(S)$ is a non-constant function of the entropy $S$. In particular, there are no special equations of state for which additional vorticity conservation laws are admitted in any even dimension, and no non-trivial vorticity conservation laws are admitted in any odd dimension $n>1$. 
The proof of this classification theorem will be given in Appendix $\mathrm{B}$.

The even-dimensional vorticity conservation law (3.30) can be rewritten through integration by parts via the identities

$$
\varpi=\mathbf{w} \cdot \boldsymbol{\omega}=\mathbf{w} \cdot(\nabla \wedge \mathbf{u}), \quad \nabla \cdot \mathbf{w}=0 .
$$

This yields the equivalent conserved integral

$$
\frac{d}{d t} \int_{V(t)} \mathbf{w} \cdot(\mathbf{u} \wedge \nabla f(S)) d^{n} x=\int_{\partial V(t)}\left(\frac{1}{2}|\mathbf{u}|^{2} \nabla f(S)-e_{S} \nabla F(S)\right) \cdot(\mathbf{w} \cdot \hat{\mathbf{n}}) d^{n-1} \sigma
$$

where $n=2 m$. We then see that the case $f(S)=S$ has the form of a circulatory entropy

$$
\frac{d}{d t} \int_{V(t)} \mathbf{w} \cdot(\mathbf{u} \wedge \nabla S) d^{n} x=\int_{\partial V(t)}\left(\frac{1}{2}|\mathbf{u}|^{2}-e_{S} S\right) \mathbf{w} \cdot(\nabla S \wedge \hat{\mathbf{n}}) d^{n-1} \sigma
$$

which vanishes whenever the fluid flow is either irrotational, i.e. $\boldsymbol{\omega}=0$ and thus $\mathbf{w}=$ $*\left(\boldsymbol{\omega}^{m-1}\right)=0$, or isentropic, i.e. $S=$ const. and thus $\nabla S=0$. Hence, in the general case, the conserved integral (3.32) can be understood to arise from conservation of circulatory entropy (3.33) by the freedom to functionally redefine the entropy $S$ to $\tilde{S}=f(S)$.

To conclude, we point out that the respective transport equations for the vorticity (3.29) in even and odd dimensions each yield locally trivial conservation laws (therefore falling outside of Theorem 3.1) in which

$$
T=*\left(\boldsymbol{\omega}^{m}\right)=\nabla \cdot *\left(\mathbf{u} \wedge \boldsymbol{\omega}^{m-1}\right)=\operatorname{div} \Theta
$$

is a spatial divergence. In particular, in even dimensions $n=2 m$, we see the transport equation (3.20) for $\varpi$ takes the form of a conservation law (2.8) given by the conserved density $T=\varpi=\nabla \cdot(\mathbf{w} \cdot \mathbf{u})$, with the moving spatial flux $\boldsymbol{\xi}=-m e_{S} \mathbf{w} \cdot \nabla S$ obtained from the identity (3.23). We note this is precisely the local conservation law that underlies the conserved integral (3.30) when the fluid flow is isentropic. Similarly, in odd dimensions $n=2 m+1(m \geq 1)$, by combining the transport equation (3.6) for $\varpi$ with the curl expression (3.11), we obtain a conservation law (2.8) where $T=\varpi=\nabla \cdot(\mathbf{W} \cdot \mathbf{u})$ is a conserved vector-density and $\boldsymbol{\xi}=-\mathbf{u} \otimes \varpi-m e_{S} \mathbf{W} \cdot \nabla S$ is the moving spatial tensor-flux.

\section{Constants of motion}

A fluid conservation law in integral form (2.9) on a domain $V(t) \subset \mathbb{R}^{n}$ moving with the fluid yields a constant of motion

$$
\frac{d}{d t} \int_{V(t)} T d^{n} x=0
$$

if the net flux through the moving domain boundary $\partial V(t)$ is zero for all solutions of the Eulerian fluid equations (2.2)-(2.4). This condition has an equivalent formulation as a local continuity equation in the form (2.8) such that the moving flux vector $\boldsymbol{\xi}$ is divergence free in the domain $V(t)$. Thus, a conserved density $T$ determines a constant of motion (4.1) if and only if

$$
\mathcal{D}_{t} T+D_{\mathbf{x}} \cdot(\mathbf{u} T)=0 .
$$

The resulting conserved integral (4.1) will be called a moving-domain constant of motion provided the conserved density is non-trivial, i.e. if $T$ is not a spatial divergence $\operatorname{div} \Theta$ such that the vector $\boldsymbol{\Theta}$ is a local function of $t, \mathbf{x}, \mathbf{u}, \rho, S$, and $\mathbf{x}$-derivatives of $\mathbf{u}, \rho, S$. 
Our classification of non-trivial kinematic and vorticity conservation laws in Theorems 2.1 and 3.1 provides an immediate corresponding classification of moving-domain constants of motion of kinematic form (2.24) and vorticity form (3.28)-(3.29).

Proposition 4.1. For compressible fluid flow in $n>1$ dimensions, the only kinematic constants of motion

$$
\frac{d}{d t} \int_{V(t)} T(t, \mathbf{x}, \mathbf{u}, \rho, S) d^{n} x=0
$$

on an arbitrary moving domain $V(t)$ transported by the fluid consist of mass

$$
\int_{V(t)} \rho d^{n} x
$$

and generalized entropy

$$
\int_{V(t)} \rho f(S) d^{n} x
$$

where $f$ is any non-constant function of $S$. These constants of motion hold for all equations of state (2.1).

In particular, no additional kinematic constants of motion arise for any special (nonisentropic or isentropic) equation of state or in any special dimension.

Proposition 4.2. There are no vorticity constants of motion

$$
\frac{d}{d t} \int_{V(t)} T(\rho, S, \mathbf{u}, \varpi) d^{n} x=0 \text { if } n=2 m \quad \text { or } \quad \frac{d}{d t} \int_{V(t)} T(\rho, S, \mathbf{u}, \varpi) d^{n} x=0 \text { if } n=2 m+1
$$

for compressible fluid flow with any non-isentropic equation of state (2.1) in $n>1$ dimensions. In the case of isentropic equations of state (2.5), where

$$
e=\int \rho^{-2} P(\rho) d \rho \quad\left(\text { with } e_{S}=0\right)
$$

is the internal (thermodynamic) energy density, the even-dimensional enstrophy

$$
\int_{V(t)} \rho f(\varpi / \rho) d^{n} x \quad(n=2 m)
$$

given by any nonlinear function $f$ yields the only vorticity constant of motion on an arbitrary moving domain $V(t)$ transported by the fluid in $n>1$ dimensions.

In addition to the classifications given by these two Propositions, we can consider nontrivial constants of motion of the form

$$
\frac{d}{d t} \int_{\partial V(t)} \Theta \cdot \hat{\mathbf{n}} d^{n-1} \sigma=0
$$

holding formally for all solutions of the fluid equations (2.2)-(2.4), where $\partial V(t)$ is the moving boundary of a domain $V(t)$ transported by the fluid. Through the divergence theorem, any conserved integral (4.9) arises from a locally trivial conserved density $T=\operatorname{div} \Theta$ satisfying the moving-flux condition (4.2) in the domain $V(t)$. In particular, a vector function $\Theta$ yields a non-trivial conserved integral (4.9) if and only if

$$
\mathcal{D}_{t} \boldsymbol{\Theta}+\mathbf{u}(\nabla \cdot \boldsymbol{\Theta})=D_{\mathbf{x}} \cdot \Psi
$$


holds for some antisymmetric tensor function $\boldsymbol{\Psi}$ in terms of $t, \mathbf{x}, \mathbf{u}, \rho, S$, and $\mathbf{x}$-derivatives of $\mathbf{u}, \rho, S$. If $\boldsymbol{\Theta}$ is not identically divergence-free, the resulting non-trivial conserved integral (4.9) will be called a moving-boundary constant of motion. We remark that the corresponding local continuity equation (4.10) can be viewed as a special type of lower-degree conservation law [6].

For isentropic fluid flow, a well-known example of a moving-boundary constant of motion is given by Kelvin's circulation theorem [18] in $n=2$ dimensions which states the circulation of the fluid velocity around any closed curve transported in the fluid is a constant of the fluid motion. The circulation integral has the form

$$
\oint_{C(t)} \mathbf{u} \cdot d \mathbf{s}=\oint_{C(t)} \mathbf{u} \cdot * \hat{\mathbf{n}} d \sigma=\oint_{C(t)} \boldsymbol{\epsilon} \cdot(\mathbf{u} \wedge \hat{\mathbf{n}}) d \sigma=-\int_{V(t)} \varpi d^{2} x
$$

related to the two-dimensional vorticity scalar $\varpi=\boldsymbol{\epsilon} \cdot \boldsymbol{\omega}=*(\nabla \wedge \mathbf{u})$ (defined in terms of the spatial orientation tensor $\boldsymbol{\epsilon}$ in $\mathbb{R}^{2}$ ), where $C(t)$ is any closed curve bounding a domain $V(t)$ transported by the fluid in $\mathbb{R}^{2}, * \hat{\mathbf{n}}$ is a unit tangent vector along $C(t)$ or equivalently $\hat{\mathbf{n}}$ is a unit normal vector, and $d \sigma$ is the arclength element for $C(t)$. As shown in [5], the circulation (4.11) has a generalization to all even dimensions $n=2 m(m>1)$ :

$$
\oint_{\partial V(t)}\left(\mathbf{u} \wedge \boldsymbol{\omega}^{m-1}\right) \cdot d \mathbf{A}=\int_{\partial V(t)}\left(\mathbf{u} \wedge \boldsymbol{\omega}^{m-1}\right) \cdot * \hat{\mathbf{n}} d^{2 m-1} \sigma=-\int_{V(t)} \varpi d^{2 m} x .
$$

This integral defines a constant of the fluid motion for isentropic fluid flow, where $d \mathbf{A}=$ $* \hat{\mathbf{n}} d^{2 m-1} \sigma$ denotes the surface element for the moving-boundary hypersurface $\partial V(t)$ in even dimensions $n=2 m$ analogously to $d \mathbf{s}=* \hat{\mathbf{n}} d \sigma$ for moving-boundary curves in two dimensions.

The natural question now arises as to whether the transport equations (3.20) and (3.6) for the vorticity (3.29) in any even or odd dimension $n>1$ yield a non-trivial constant of motion (4.9) when the fluid flow is non-isentropic. Our classification proof for vorticity conservation laws in Theorem 3.1 actually settles this question in a slightly more general form.

Proposition 4.3. For compressible fluid flow with any non-isentropic equation of state (2.1) in $n>1$ dimensions, there are no moving-boundary constants of motion (4.9) whose corresponding local continuity equation (4.2) for $T=\operatorname{div} \Theta$ is of vorticity form (3.28)-(3.29). In the case of isentropic equations of state (2.5), the only moving-boundary constant of motion with such a form is given by the generalized circulation (4.12) in all even dimensions.

In particular, for non-isentropic fluid flow, the vorticity transport equations written in the moving-boundary form (3.34) show that

$$
\frac{d}{d t} \oint_{\partial V(t)}\left(\mathbf{u} \wedge \boldsymbol{\omega}^{m-1}\right) \cdot d \mathbf{A}=m \oint_{\partial V(t)} e_{S}\left(\nabla S \wedge \boldsymbol{\omega}^{m-1}\right) \cdot d \mathbf{A}
$$

in even dimensions $n=2 m$, while in odd dimensions $n=2 m+1$

$$
\frac{d}{d t} \oint_{\partial V(t)}\left(\mathbf{u} \wedge \boldsymbol{\omega}^{m-1}\right) \cdot d \mathbf{A}=m \oint_{\partial V(t)} e_{S}\left(\nabla S \wedge \boldsymbol{\omega}^{m-1}\right) \cdot d \mathbf{A}-\oint_{\partial V(t)} \mathbf{u} \boldsymbol{\omega}^{m} \cdot d \mathbf{A}
$$

with $d \mathbf{A}=* \hat{\mathbf{n}} d^{2 m-1} \sigma$, where $e_{S} \not \equiv 0$ is a non-constant function of $\rho$ for any non-isentropic equation of state (2.1). 


\section{HAMiltonian CORRESPONDENCE}

A Hamiltonian formulation for the non-isentropic compressible Euler equations (2.1)-(2.4) in $n>1$ dimensions (cf [16]) is given by

$$
\partial_{t}\left(\begin{array}{c}
\mathbf{u} \\
\rho \\
S
\end{array}\right)=\mathcal{H}\left(\begin{array}{c}
\delta E / \delta \mathbf{u} \\
\delta E / \delta \rho \\
\delta E / \delta S
\end{array}\right), \quad E=\frac{1}{2} \rho|\mathbf{u}|^{2}+\rho e(\rho, S)=\rho\left(\frac{1}{2}|\mathbf{u}|^{2}+\int \rho^{-2} P(\rho, S) d \rho\right),
$$

in terms of the Hamiltonian operator

$$
\mathcal{H}=\left(\begin{array}{ccc}
\rho^{-1}(\nabla \wedge \mathbf{u}) \cdot & -\nabla & \rho^{-1} \nabla S \\
-\nabla \cdot & 0 & 0 \\
-\left(\rho^{-1} \nabla S\right) \cdot & 0 & 0
\end{array}\right)
$$

where $E$ is the energy density of the fluid. This operator (5.2) determines a Poisson bracket

$$
\begin{aligned}
& \{\mathcal{F}, \mathcal{G}\}_{\mathcal{H}}=\int\left(\begin{array}{lll}
\delta F / \delta \mathbf{u} & \delta F / \delta \rho & \delta F / \delta S
\end{array}\right) \mathcal{H}\left(\begin{array}{c}
\delta G / \delta \mathbf{u} \\
\delta G / \delta \rho \\
\delta G / \delta S
\end{array}\right) d^{n} x \\
& =\int \rho^{-1}(\nabla \wedge \mathbf{u}) \cdot(\delta F / \delta \mathbf{u} \wedge \delta G / \delta \mathbf{u})+\delta G / \delta \mathbf{u} \cdot\left(\nabla \delta F / \delta \rho-\left(\rho^{-1} \delta F / \delta S\right) \nabla S\right) \\
& -\delta F / \delta \mathbf{u} \cdot\left(\nabla \delta G / \delta \rho-\left(\rho^{-1} \delta G / \delta S\right) \nabla S\right) d^{n} x
\end{aligned}
$$

satisfying (modulo divergence terms) antisymmetry and the Jacobi identity [19], for arbitrary functionals $\mathcal{F}=\int F d^{n} x$ and $\mathcal{G}=\int G d^{n} x$ where $F$ and $G$ are functions of $t, \mathbf{x}, \mathbf{u}, \rho, S$, and $\mathbf{x}$-derivatives of $\mathbf{u}, \rho, S$. Here $\delta / \delta \mathbf{u}, \delta / \delta \rho, \delta / \delta S$ denote variational derivatives, which respectively coincide with the spatial Euler operators $E_{\mathbf{u}}, E_{\rho}, E_{S}$ when acting on functions that do not contain time derivatives of $\mathbf{u}, \rho, S$.

Similarly to the isentropic case described in [5], the Hamiltonian operator $\mathcal{H}$ gives rise to an explicit mapping

$$
-\mathcal{H}\left(\begin{array}{c}
\delta T / \delta \mathbf{u} \\
\delta T / \delta \rho \\
\delta T / \delta S
\end{array}\right)=\widehat{\mathbf{X}}\left(\begin{array}{c}
\mathbf{u} \\
\rho \\
S
\end{array}\right)=\left(\begin{array}{c}
\hat{\boldsymbol{\eta}} \\
\hat{\eta} \\
\hat{\phi}
\end{array}\right)
$$

which produces infinitesimal symmetries $\widehat{\mathbf{X}}=\hat{\boldsymbol{\eta}}\rfloor \partial_{\mathbf{u}}+\hat{\eta} \partial_{\rho}+\hat{\phi} \partial_{S}$ of the non-isentropic Euler equations from conserved densities $T$. In particular, these components of the symmetry generator

$\hat{\boldsymbol{\eta}}=-\rho^{-1}(\nabla \wedge \mathbf{u}) \cdot \delta T / \delta \mathbf{u}+\nabla \delta T / \delta \rho-\left(\rho^{-1} \delta T / \delta S\right) \nabla S, \quad \hat{\eta}=\nabla \cdot \delta T / \delta \mathbf{u}, \quad \hat{\phi}=\rho^{-1} \nabla S \cdot \delta T / \delta \mathbf{u}$

will satisfy the infinitesimal invariance equations [19, 10]

$$
\begin{aligned}
& D_{t} \hat{\boldsymbol{\eta}}+\mathbf{u} \cdot \nabla \hat{\boldsymbol{\eta}}+\hat{\boldsymbol{\eta}} \cdot \nabla \mathbf{u}-\rho^{-2} \hat{\eta} \nabla P+\rho^{-1} \nabla\left(P_{\rho} \hat{\eta}+P_{S} \hat{\phi}\right)=0, \\
& D_{t} \hat{\eta}+\nabla \cdot \mathbf{u} \hat{\eta}+\rho \nabla \cdot \hat{\boldsymbol{\eta}}=0, \quad D_{t} \hat{\phi}+\hat{\boldsymbol{\eta}} \cdot \nabla S+\mathbf{u} \cdot \nabla \hat{\phi}=0
\end{aligned}
$$

for all solutions of the non-isentropic Euler equations (2.1)-(2.4).

Evaluating the mapping (5.4) -(5.5) for the kinematic and vorticity conserved densities given by the conserved integrals in Theorems 2.1 and 3.1, we obtain the following two tables 
of symmetries, all of which can be written as point transformations $\left.\left.\mathbf{X}=\tau \partial_{t}+\boldsymbol{\xi}\right\rfloor \mathbf{x}+\boldsymbol{\eta}\right\rfloor \partial_{\mathbf{u}}+$ $\eta \partial_{\rho}+\phi \partial_{S}$ given by

$$
\hat{\boldsymbol{\eta}}=\boldsymbol{\eta}-\tau \partial_{t} \mathbf{u}-\boldsymbol{\xi} \cdot \nabla \mathbf{u}, \quad \hat{\eta}=\eta-\tau \partial_{t} \rho-\boldsymbol{\xi} \cdot \nabla \rho, \quad \hat{\phi}=\phi-\tau \partial_{t} S-\boldsymbol{\xi} \cdot \nabla S,
$$

where $\boldsymbol{\eta}, \eta, \phi$ are functions of $t, \mathbf{x}, \rho, S, \mathbf{u}$, while $\tau, \boldsymbol{\xi}$ are functions only of $t, \mathbf{x}$.

For general non-isentropic equations of state (2.1):

\begin{tabular}{|c|c|c|c|}
\hline Conserved Density $T$ & Conservation Law & Symmetry $\mathbf{X}$ & Description \\
\hline$\rho$ & Mass & 0 & Nil \\
$\rho \mathbf{u}$ & Momentum & $\partial_{\mathbf{x}}$ & Space translations \\
$\rho \mathbf{u} \wedge \mathbf{x}$ & Angular Momentum & $\mathbf{x} \wedge \partial_{\mathbf{x}}+\mathbf{u} \wedge \partial_{\mathbf{u}}$ & Rotations \\
$\rho(t \mathbf{u}-\mathbf{x})$ & Galilean Momentum & $t \partial_{\mathbf{x}}+\partial_{\mathbf{u}}$ & Galilean boosts \\
$\frac{1}{2} \rho|\mathbf{u}|^{2}+\rho e$ & Energy & $\partial_{t}$ & Time translation \\
$\rho S$ & Volumetric Entropy & 0 & Nil \\
$\varpi S \equiv *\left(\boldsymbol{\omega}^{m-1} \wedge \mathbf{u} \wedge \nabla S\right)$ & Circulatory Entropy $(n=2 m)$ & 0 & Nil \\
\hline
\end{tabular}

Here $e$ is the internal (thermodynamic) energy density (2.17). Note the entropy (per unit mass) $S$ can be replaced by an arbitrary function $f(S)$.

For polytropic equations of state (2.25):

\begin{tabular}{|c|c|c|c|}
\hline Conserved Density $T$ & Conservation Law & Symmetry X & Description \\
\hline$t E-\frac{1}{2} \rho(\mathbf{u} \cdot \mathbf{x})$ & Similarity Energy & $\begin{array}{c}\left.t \partial_{t}+\frac{1}{2} \mathbf{x}\right\rfloor \partial_{\mathbf{x}} \\
-\frac{1}{2} \mathbf{u} \partial_{\mathbf{u}}-\frac{1}{2} n \rho \partial_{\rho} \\
\left.t^{2} \partial_{t}+t \mathbf{x}\right\rfloor \partial_{\mathbf{x}} \\
-(t \mathbf{u}-\mathbf{x}) \partial_{\mathbf{u}}-n t \rho \partial_{\rho}\end{array}$ & Similarity Scaling \\
$t^{2} E-t \rho(\mathbf{u} \cdot \mathbf{x})+\frac{1}{2} \rho|\mathbf{x}|^{2}$ & Galilean Energy & Galilean Dilation \\
\hline
\end{tabular}

Here $E=\rho\left(\frac{1}{2}|\mathbf{u}|^{2}+e\right)=\frac{1}{2} \rho|\mathbf{u}|^{2}+\frac{1}{2} n \kappa(S) \rho^{1+2 / n}$ is the polytropic energy density.

As none of these symmetry generators $\mathbf{X}$ contain the pressure $p$, we see that the symmetry structure produced via the Hamiltonian mapping (5.5) for the non-isentropic Euler equations (2.1) -(2.4) is the same as in the isentropic case presented in [5]. This structure can be geometrically summarized as follows.

Recall, the Killing equation on $\mathbb{R}^{n}$ is given by

$$
\mathcal{L}_{\zeta} \mathbf{g}=0
$$

for a vector $\boldsymbol{\zeta}(\mathbf{x})$, where $\mathcal{L}$ denotes the Lie derivative and $\mathbf{g}$ is the Euclidean metric tensor. Also recall, a vector $\boldsymbol{\zeta}(\mathbf{x})$ is irrotational on $\mathbb{R}^{n}$ if $\nabla \wedge \boldsymbol{\zeta}=0$.

Proposition 5.1. (i) For a general equation of state (2.1), the Hamiltonian symmetries corresponding to the kinematic conserved densities for energy (2.15), momentum (2.12), angular momentum (2.13) and Galilean momentum 2.14) comprise the generators of the Galilean group in $n>1$ dimensions given by

$$
\left.\left.\left.\left.\mathbf{X}=\partial_{t}, \quad \mathbf{X}=\boldsymbol{\zeta}\right\rfloor \partial_{\mathbf{x}}+\frac{1}{2} \mathbf{u} \cdot(\nabla \wedge \boldsymbol{\zeta})\right\rfloor \partial_{\mathbf{u}}, \quad \mathbf{X}=t \boldsymbol{\chi}\right\rfloor \partial_{\mathbf{x}}+\boldsymbol{\chi}\right\rfloor \partial_{\mathbf{u}},
$$

in terms of solutions $\boldsymbol{\zeta}(\mathbf{x})$ of the Killing equation, and irrotational solutions $\boldsymbol{\chi}(\mathbf{x})$ of the Killing equation. (ii) The additional Hamiltonian symmetries, consisting of the similarity scaling and Galilean dilation which correspond to the generalized energy densities (2.20) and (2.21) in the case of a polytropic equation of state (2.25), generate an extension of the Galilean group given by

$$
\begin{gathered}
\left.\left.\mathbf{X}=\partial_{t}, \quad \mathbf{X}=\lambda t \partial_{t}+\boldsymbol{\zeta}\right\rfloor \partial_{\mathbf{x}}-\frac{1}{2} \lambda n \rho \partial_{\rho}+\frac{1}{2}(\mathbf{u} \cdot(\nabla \wedge \boldsymbol{\zeta})-\lambda \mathbf{u})\right\rfloor \partial_{\mathbf{u}} \\
\left.\left.\mathbf{X}=\frac{1}{2} \sigma t^{2} \partial_{t}+t \boldsymbol{\chi}\right\rfloor \partial_{\mathbf{x}}-\frac{1}{2} \sigma n t \rho \partial_{\rho}+\left(\boldsymbol{\chi}-\frac{1}{2} t \sigma \mathbf{u}\right)\right\rfloor \partial_{\mathbf{u}},
\end{gathered}
$$


where the vector $\boldsymbol{\zeta}(\mathbf{x})$ is the solution of the homothetic Killing equation $\mathcal{L}_{\zeta} \mathbf{g}=\lambda \mathbf{g}, \lambda=$ const., and the vector $\boldsymbol{\chi}(\mathbf{x})$ is the irrotational solution of the homothetic Killing equation $\mathcal{L}_{\chi} \mathbf{g}=\sigma \mathbf{g}$, $\sigma=$ const. .

In contrast, the conserved densities for mass (2.11), generalized entropy (2.18), and circulatory entropy (3.32) (or equivalently (3.30)) are mapped into the trivial symmetry $\mathbf{X}=0$. These conserved densities comprise all of the Hamiltonian Casimirs for the non-isentropic Euler equations (2.1)-(2.4) in $n>1$ dimensions.

\section{Gas Dynamics Conservation Laws}

The Euler equations (2.1)-(2.4) for non-isentropic compressible fluid flow have an equivalent formulation in which the equation of state (2.1) is inverted to give entropy as a function of pressure and density, $S=S(p, \rho)$ with $\partial S / \partial p \not \equiv 0$. The entropy transport equation (2.4) then becomes

$$
\partial_{t} p+\mathbf{u} \cdot \nabla p+F(p, \rho) \nabla \cdot \mathbf{u}=0
$$

in terms of the function

$$
F(p, \rho)=-\rho \frac{\partial S / \partial \rho}{\partial S / \partial p}
$$

which replaces the equation of state (2.1). For any specific choice of $F(p, \rho)$, the entropy function $S(p, \rho)$ can be recovered as a solution of the linear equation

$$
\rho S_{\rho}+F(p, \rho) S_{p}=0
$$

In particular, we can identify $S(p, \rho)$ with the constant of integration arising from the characteristic ODE

$$
\frac{d p}{d \rho}=F(p, \rho) / \rho
$$

Note that the simple state function

$$
F=\gamma p, \quad \gamma=\text { const. }
$$

corresponds to a polytropic equation of state (2.25) which is equivalent to an entropy function of the form $S=S\left(p / \rho^{\gamma}\right)$.

The resulting formulation of compressible fluid flow thus consists of the dynamical equations (6.1), (2.2), (2.3) for pressure $p$, velocity $\mathbf{u}$, and density $\rho$, respectively. These are precisely the equations of adiabatic gas dynamics [26]. Physically, the state function $F(p, \rho)$ is then related to the sound speed $c$ in the gas by $F / \rho=c^{2}$ as seen from equation (6.4). The state function also determines the internal energy density $e$ of the gas from the thermodynamic relations $d e=-p d(1 / \rho)=e_{\rho} d \rho+e_{p} d p$ combined with equation (6.4), which yields

$$
\rho e_{\rho}+F(p, \rho) e_{p}=\frac{p}{\rho}
$$

for the function $e(p, \rho)$. Note the homogeneous part of this linear equation (6.6) involves the same linear differential operator $\rho \partial_{\rho}+F(p, \rho) \partial_{p}$ appearing in the entropy equation (6.3) , so thus the general homogeneous solution of equation (6.6) is given by $f(S(p, \rho))$ in terms of any particular solution of equation (6.3), where $f$ is an arbitrary function of $S(p, \rho)$.

Since the dynamical pressure equation (6.4) is related to the entropy transport equation (2.4) by an invertible change of variables $p=P(\rho, S) \leftrightarrow S=S(p, \rho)$ (i.e. a point transformation) when $S_{p} \not \equiv 0$ or $P_{S} \not \equiv 0$, there is consequently a one-to-one correspondence between 
the local conservation law structure of the gas dynamics equations (6.1), (6.2), (2.2), (2.3) and the non-isentropic Euler equations (2.1)-(2.4), as shown by general results in [11, 10]. A complete classification of kinematic and vorticity conservation laws for gas dynamics in $n>1$ dimensions now follows as a corollary to Theorems 2.1 and 3.1.

Theorem 6.1. (i) For a general state function $F(p, \rho)$, all gas dynamics conserved densities of kinematic form $T(t, \mathbf{x}, \mathbf{u}, \rho, p)$ in any dimension $n>1$ are given by a linear combination of the conservation laws for mass (2.11), momentum (2.12), angular momentum (2.13), Galilean momentum (2.14), plus energy (2.15) and volumetric entropy (2.19) where $e=$ $e(p, \rho)$ is any non-homogeneous solution of the thermodynamic energy equation (6.6) and $S=S(p, \rho)$ is the general solution of the entropy equation (6.3). (ii) The only state function $F(p, \rho)$ for which additional kinematic conserved densities arise is the polytropic case (6.5) with dimension-dependent coefficient $\gamma=1+2 / n$. The admitted conservation laws consist of the similarity energy (2.20) and the Galilean energy (2.21) given in terms of the polytropic energy density (2.23).

Theorem 6.2. (i) For a general state function $F(p, \rho)$, all gas dynamics conserved densities of vorticity form $T(\rho, p, \mathbf{u}, \varpi)$ if $n=2 m$ or $T(\rho, p, \mathbf{u}, \varpi)$ if $n=2 m+1$ (with $m \geq 1$ ) consist of the conservation law for circulatory entropy (3.33) in even dimensions, as expressed in terms of $\mathbf{w}=*\left(\boldsymbol{\omega}^{m-1}\right), \nabla S=S_{p}\left(\nabla p-\rho^{-1} F(p, \rho) \nabla \rho\right)$, and $e_{S}=e_{p} / S_{p}$, where $S=S(p, \rho)$ is the general solution of the entropy equation (6.3), $e=e(p, \rho)$ is any non-homogeneous solution of the thermodynamic energy equation (6.6), and $\mathbf{w}$ is an antisymmetric tensor related to the vorticity scalar $\varpi$ by the identities (3.31). (ii) There are no state functions $F(p, \rho)$ for which additional vorticity conserved densities arise in any even or odd dimension $n>1$.

\section{Multipliers}

In index notation, the Euler equations for inviscid non-isentropic compressible fluid flow on $\mathbb{R}^{n}$ in Cartesian coordinates $x^{i}(i=1, \ldots, n)$ are given by

$$
\begin{gathered}
u_{t}^{i}+u^{j} u_{, j}^{i}+\rho^{-1} p_{,}^{i}=0, \\
\rho_{t}+\left(\rho u^{i}\right)_{, i}=0, \\
S_{t}+u^{i} S_{, i}=0,
\end{gathered}
$$

together with the equation of state

$$
p=P(\rho, S), \quad P_{S} \not \equiv 0 .
$$

Similarly to the isentropic case studied in [5], any local conservation law $\mathcal{D}_{t} T+D_{i} X^{i}=0$ that holds formally for all solutions of these equations (7.1)-(7.4) can be written in an equivalent characteristic form

$$
D_{t} T+D_{i} \tilde{X}^{i}=\left(u_{t}^{i}+u^{j} u_{, j}^{i}+\rho^{-1} P_{\rho} \rho_{,}^{i}+\rho^{-1} P_{S} S_{,}^{i}\right) Q_{i}+\left(\rho_{t}+\left(\rho u^{i}\right)_{, i}\right) Q+\left(S_{t}+u^{i} S_{, i}\right) R
$$

in terms of the multipliers

$$
Q_{i}^{u}=E_{u^{i}}(T), \quad Q^{\rho}=E_{\rho}(T), \quad Q^{S}=E_{S}(T) .
$$

In this formulation $u^{i}, \rho, S$ are given by arbitrary functions of $t$ and $x^{i}$, with $\tilde{X}^{i}$ differing from $X^{i}$ by terms that are linear homogeneous in the fluid equations (17.1)-(17.3) and their total spatial derivatives. Since the spatial Euler operators $E_{u^{i}}, E_{\rho}, E_{S}$ annihilate any spatial 
divergence $D_{i} \Theta^{i}$, there is then a one-to-one correspondence between nontrivial conserved densities $T$ (modulo spatial divergences) and non-zero multipliers $\left\{Q_{i}^{u}, Q^{\rho}, Q^{S}\right\}$.

Necessary and sufficient equations for determining multipliers (7.6) are given by applying variational derivative operators $\delta / \delta u^{i}, \delta / \delta \rho, \delta / \delta S$ to the characteristic equation (7.5), yielding a linear homogeneous polynomial system in $u_{t}^{i}, \rho_{t}, S_{t}, u_{t, j}^{i}, \rho_{t, j}, S_{t, j}$, etc. whose coefficients must separately vanish. The resulting determining equations for $Q_{i}^{u}, Q^{\rho}, Q^{S}$ consist of the adjoint of the determining equations for symmetries (5.6), plus additional integrablity equations (Helmholtz conditions) for $Q_{i}^{u}, Q^{\rho}, Q^{S}$ to have the variational form (7.6). Thus, multipliers can be characterized as adjoint-symmetries that have a variational form [2, 3, 4, 10]. Moreover, the corresponding conserved density $T$ (up to an arbitrary spatial divergence) can be constructed explicitly from the multipliers $Q_{i}^{u}, Q^{\rho}, Q^{S}$ by means of homotopy integral formulas [19, 4, 10] or by an algebraic scaling formula [1] based on invariance of the fluid equations under dilations $t \rightarrow \lambda t, x^{i} \rightarrow \lambda x^{i}$. The determination of multipliers and hence of conservation laws is thereby reduced to an adjoint version of the determination of symmetries.

To conclude, in the following two tables we list the multipliers for, firstly, the non-isentropic kinematic conservation laws (2.11) -(2.15) and the non-isentropic vorticity conservation laws (3.30) and (equivalently) (3.32) -(3.33), all of which hold for a general equation of state (2.1); and, secondly, the extra non-isentropic kinematic conservation laws (2.20)-(2.21) holding only for the distinguished polytropic equation of state (2.25).

\begin{tabular}{|c|c|c|c|c|}
\hline Conserved density $T$ & Description & $Q_{i}^{u}=\delta T / \delta u^{i}$ & $Q^{\rho}=\delta T / \delta \rho$ & $Q^{S}=\delta T / \delta S$ \\
\hline$\rho$ & Mass & 1 & 0 & 0 \\
$\rho u^{k}$ & Momentum & $\rho \delta_{i}^{k}$ & $u^{k}$ & 0 \\
$\rho\left(u^{j} x^{k}-u^{k} x^{j}\right)$ & Angular momentum & $\rho\left(x^{k} \delta_{i}^{j}-x^{j} \delta_{i}^{k}\right)$ & $u^{j} x^{k}-u^{k} x^{j}$ & 0 \\
$\rho\left(t u^{k}-x^{k}\right)$ & Galilean momentum & $\rho t \delta_{i}^{k}$ & $t u^{k}-x^{k}$ & 0 \\
$\rho\left(\frac{1}{2} u^{k} u_{k}+e\right)$ & Energy & $\rho u_{i}$ & $\frac{1}{2} u^{k} u_{k}+e+\rho e_{\rho}$ & $\rho e_{S}$ \\
$\rho S$ & Volumetric entropy & 0 & $S$ & $\rho$ \\
$\varpi S$ & Circulatory & $S,{ }^{j} \partial \varpi / \partial \omega^{i j}$ & 0 & $\varpi$ \\
$\equiv *\left(\omega^{m-1}\right)_{i j} u^{i} S_{,}{ }^{j}$ & entropy $(n=2 m)$ & & & \\
\hline
\end{tabular}

\begin{tabular}{|c|c|c|c|c|}
\hline Conserved density $T$ & Description & $Q_{i}^{u}=\delta T / \delta u^{i}$ & $Q^{\rho}=\delta T / \delta \rho$ & $Q^{S}=\delta T / \delta S$ \\
\hline$t E-\frac{1}{2} \rho u^{k} x_{k}$ & Similarity Energy & $\rho\left(t u_{i}-\frac{1}{2} x_{i}\right)$ & $\frac{1}{2} u^{k}\left(t u_{k}-x_{k}\right)$ & $t e_{S}$ \\
& & $+t\left(1+\frac{1}{2}\right) n \kappa(S) \rho^{2 / n}$ & \\
$t^{2} E-\rho\left(t u^{k}-\frac{1}{2} x^{k}\right) x_{k}$ & Galilean Energy & $t \rho\left(t u_{i}-x_{i}\right)$ & $\frac{1}{2}\left(t u^{k}-x^{k}\right)\left(t u_{k}-x_{k}\right)$ & $t^{2} e_{S}$ \\
& & & $+t^{2}\left(1+\frac{1}{2} n\right) \kappa(S) \rho^{2 / n}$ & \\
\hline
\end{tabular}

Here $E=\rho\left(\frac{1}{2} u^{k} u_{k}+e\right)=\frac{1}{2} \rho u^{k} u_{k}+\frac{1}{2} n \kappa(S) \rho^{1+2 / n}$ is the polytropic energy density, in terms of the internal energy density $e=\frac{1}{2} n \kappa(S) \rho^{2 / n}$.

\section{Appendix A. Proof of Theorem 2.1 and Proposition 4.1}

The time derivative of a kinematic conserved density $T\left(t, x^{i}, \rho, u^{i}, S\right)$ is given by

$$
\mathcal{D}_{t} T=-T_{S} u^{i} S_{, i}-T_{\rho}\left(u^{i} \rho_{, i}+\rho u_{, i}^{i}\right)-T_{u^{i}}\left(u^{j} u^{i}{ }_{, j}+\rho^{-1} P_{,}^{i}\right)+T_{t} .
$$

By applying each Euler operator $E_{\rho}, E_{S}, E_{u^{i}}$ to (A.1), we get three linear inhomogeneous expressions in $u^{j}{ }_{j}, u^{j}{ }_{i}, \rho_{,}{ }^{j}, S_{,}{ }^{j}$, whose coefficients must separately vanish. This yields the 
system of determining equations

$$
\begin{gathered}
\rho^{-1} P_{S} T_{u^{i} x_{i}}+T_{S x^{i}} u^{i}+T_{t S}=0, \\
\rho T_{\rho x^{i}}+T_{u^{i} x^{j}} u^{j}+T_{t u^{i}}=0, \\
T_{\rho x^{i}} u^{i}+\rho^{-1} P_{\rho} T_{u^{i} x_{i}}+T_{t \rho}=0, \\
\left(\rho T_{\rho S}-T_{S}\right) \delta_{i j}-\rho^{-1} P_{S} T_{u^{i} u^{j}}=0, \\
\rho^{2} T_{\rho \rho} \delta_{i j}-P_{\rho} T_{u^{i} u^{j}}=0, \\
\left(\rho T_{u^{i} \rho}-T_{u^{i}}\right) P_{S}-\rho P_{\rho} T_{u^{i} S}=0, \\
\rho T_{u^{i} \rho}-T_{u^{i}}=0,
\end{gathered}
$$

to be solved for $T$. Here $\delta_{i j}=\delta_{(i j)} \leftrightarrow \mathbf{g}$ denotes the Cartesian components of the Euclidean metric tensor on $\mathbb{R}^{n}$.

We start by integrating equation (A.8) with respect to $\rho$ and $u^{i}$, yielding

$$
T=\rho f\left(t, x^{i}, u^{i}, S\right)+g\left(t, x^{i}, \rho, S\right) .
$$

Now substituting (A.9) into (A.7) we get $f_{u^{i} S}=0$ and then integrating gives

$$
f=\tilde{f}\left(t, x^{i}, u^{i}\right)
$$

where an integration constant $c\left(t, x^{i}, S\right)$ has been dropped since we can absorb $\rho c\left(t, x^{i}, S\right)$ into the term $g\left(t, x^{i}, \rho, S\right)$ in (A.9).

Next, substituting (A.9) and (A.10) into both (A.6) and (A.5), we obtain

$$
\begin{gathered}
\rho g_{\rho \rho} \delta_{i j}-\tilde{f}_{u^{i} u^{j}} P_{\rho}=0, \\
\left(\rho g_{\rho S}-g_{S}\right) \delta_{i j}-\tilde{f}_{u^{i} u^{j}} P_{S}=0 .
\end{gathered}
$$

Separation of (A.11) with respect to $u^{i}$ yields the equations

$$
\begin{gathered}
\tilde{f}_{u^{i} u^{j}}=c\left(t, x^{k}\right) \delta_{i j}, \\
g_{\rho \rho}=c\left(t, x^{k}\right) \rho^{-1} P_{\rho},
\end{gathered}
$$

where $c\left(t, x^{k}\right)$ is a separation constant. Substituting (A.13) into (A.12), we get

$$
\rho g_{\rho S}-g_{S}=c\left(t, x^{k}\right) P_{S}
$$

By writing (A.12) and (A.15) as $\left(\rho g_{\rho}-g-c P\right)_{\rho}=\left(\rho g_{\rho}-g-c P\right)_{S}=0$, then integrating with respect to $\rho$ and $S$, we obtain a first-order linear equation with respect to $\rho$,

$$
\rho g_{\rho}-g=c P-c_{1}\left(t, x^{i}\right) \text {. }
$$

Integration of (A.16) and $(\underline{\mathrm{A} .13})$ then yields

$$
\tilde{f}=\tilde{c}_{j}\left(t, x^{i}\right) u^{j}+\frac{1}{2} c\left(t, x^{i}\right) u^{j} u_{j}, \quad g=\rho^{-1} c_{1}\left(t, x^{i}\right)+c\left(t, x^{i}\right) \int \rho^{-2} P(\rho, S) d \rho,
$$

after integration constants have been absorbed as before. Consequently, from (A.9) we have

$$
T=c_{1}\left(t, x^{i}\right)+c_{2}\left(t, x^{i}, S\right) \rho+\tilde{c}_{j}\left(t, x^{i}\right) \rho u^{j}+c\left(t, x^{i}\right)\left(\frac{1}{2} \rho u^{j} u_{j}+\rho e(\rho, S)\right)
$$

which gives the general solution of the determining equations (A.8), (A.7), (A.6), (A.5), with $e(\rho, S)$ given by (2.17) in terms of $P(\rho, S)$. Note the term $c_{1}$ in (A.18) is a trivial conserved density, and so we will put $c_{1}=0$. 
By substituting (A.18) into the remaining determining equations (A.4), (A.3), (A.2), we get a system of equations to be solved for $c\left(t, x^{i}\right), \tilde{c}_{j}\left(t, x^{i}\right), c_{2}\left(t, x^{i}, S\right), c_{1}\left(t, x^{i}\right)$ :

$$
\begin{gathered}
c_{x^{i}}=0, \\
\tilde{c}_{j x^{i}}+\tilde{c}_{i x^{j}}+c_{t} \delta_{i j}=0, \\
c_{2 x^{i}}+\tilde{c}_{i t}=0, \\
\tilde{c}_{i x_{i}} P_{\rho}+c_{t} e+c_{t} \rho e_{\rho}+c_{2 t}=0, \\
\tilde{c}_{i x_{i}} P_{S}+c_{t} \rho e_{S}+\rho c_{2 S t}=0, \\
c_{2 S x^{i}}=0 .
\end{gathered}
$$

To begin, we note (A.19) implies $c=c(t)$, and hence (A.20) has the form of a timedependent homothetic Killing vector equation on $\tilde{c}_{i}$. Its solution consists of a linear polynomial in $x^{i}$ given by

$$
\tilde{c}_{i}=C_{0 i}(t)+C_{1 i j}(t) x^{j}-\frac{1}{2} c^{\prime}(t) x_{i}, \quad C_{1 i j}(t)=-C_{1 j i}(t) .
$$

Then, using the trace of (A.20) in (A.22), followed by differentiating with respect to $x^{i}$, we find

$$
c_{2 t x^{i}}=0 .
$$

Integration of (A.24) and (A.26) gives

$$
c_{2}=\tilde{c}_{2}(t, S)+\hat{c}_{2}\left(x^{i}\right) .
$$

Substituting (A.27) and (A.25) into (A.21), we get

$$
\hat{c}_{2 x^{i}}=-C_{0 i}^{\prime}-C_{1 i j}^{\prime} x^{j}+\frac{1}{2} c^{\prime \prime} x_{i} .
$$

By differentiating with respect to $x^{j}$ and antisymmetrizing in $i, j$, we find $C_{1 i j}^{\prime}=0$, so thus $C_{1 i j}$ is constant. Integration of (A.28) thus yields

$$
\hat{c}_{2}=C_{2}-C_{0 i}^{\prime} x^{i}+\frac{1}{4} c^{\prime \prime} x_{i} x^{i}
$$

where $C_{2}$ is a constant. Then, differentiating (A.29) with respect to $t$, we get $-C_{0 i}^{\prime \prime} x^{i}+$ $\frac{1}{4} c^{\prime \prime \prime} x_{i} x^{i}=0$ which splits with respect to $x^{i}$ into $C_{0 i}^{\prime \prime}=0, c^{\prime \prime \prime}=0$. Hence we obtain

$$
C_{0 i}=a_{0 i}+a_{1 i} t, \quad c=b_{0}+b_{1} t+b_{2} t^{2},
$$

and, from (A.25) and (A.29),

$$
\tilde{c}_{i}=a_{0 i}+a_{1 i} t+C_{1 i j} x^{j}-\frac{1}{2}\left(b_{1}+b_{2} t\right) x_{i}, \quad \hat{c}_{2}=C_{2}-a_{1 i} x^{i}+\frac{1}{2} b_{2} x^{i} x_{i},
$$

where $a_{0 i}, a_{1 i}, b_{0}, b_{1}, b_{2}$ are constants. Now, through (A.31), we can rewrite (A.22) and (A.23) as

$$
c^{\prime}\left(-\frac{1}{2} n P+\rho e\right)_{\rho}=-\left(\rho \tilde{c}_{2 t}\right)_{\rho}, \quad c^{\prime}\left(-\frac{1}{2} n P+\rho e\right)_{S}=-\left(\rho \tilde{c}_{2 t}\right)_{S} .
$$

Integrating (A.32) with respect to $\rho$ and $S$, we obtain

$$
\left(\rho e-\frac{1}{2} n P\right) c^{\prime}+\rho \tilde{c}_{2 t}=a(t) .
$$

By considering the separation of (A.33) with respect to $\rho$, we have the following two cases.

Case $c^{\prime}=0$ : Here $c=$ const. $=b_{0}$ and hence $b_{1}=b_{2}=0$ in (A.30). Now splitting (A.33) with respect to $\rho$ yields $a=0$ and $\tilde{c}_{2 t}=0$, so thus

$$
\tilde{c}_{2}=b(S)
$$


where, through (A.27), we have absorbed an integration constant into $C_{2}$. Hence, (A.18) reduces to

$$
T=C_{2} \rho+b(S) \rho+a_{0 i} \rho u^{i}+a_{1 i} \rho\left(t u^{i}-x^{i}\right)+C_{1 i j} \rho u^{i} x^{j}+b_{0}\left(\frac{1}{2} \rho u^{i} u_{i}+\rho e(\rho, S)\right)
$$

with arbitrary constants $a_{0 i}, a_{1 i}, b_{0}, C_{1 i j}=-C_{1 j i}, C_{2}$, and an arbitrary function $b(S)$, where $e(\rho, S)$ is given by (2.17).

Case $c^{\prime} \neq 0$ : Dividing (A.33) by $\rho$, then differentiating with respect to $\rho$ and multiplying by $\rho^{2}$, we get $\rho^{2} c^{\prime}\left(e_{\rho}-\frac{1}{2} n\left(\rho^{-1} P\right)_{\rho}\right)=-a$ which separates with respect to $t$ and $S$ into the equations

$$
\begin{gathered}
\rho^{2}\left(e_{\rho}-\frac{1}{2} n\left(\rho^{-1} P\right)_{\rho}\right)=d, \\
c^{\prime} d=-a,
\end{gathered}
$$

where $d$ is a constant of separation. Substituting $e$ from (2.17) into (A.36), we obtain a firstorder linear equation with respect to $\rho,-\frac{1}{2} n \rho P_{\rho}+\left(1+\frac{1}{2} n\right) P=d$, whose general solution is given by

$$
P(\rho, S)=\kappa(S) \rho^{1+2 / n}+d /(1+n / 2) .
$$

Since we can (without loss of generality) shift the pressure $P$ by an arbitrary constant, we put $d=0$. Then, (A.37) yields $a=0$, while (2.17) becomes

$$
e(\rho, S)=\frac{1}{2} n \rho^{-1} P+\tilde{e}(S)
$$

Substituting (A.39) into (A.33), we get $c^{\prime} \tilde{e}(S)+\tilde{c}_{2 t}=0$ which gives

$$
c \tilde{e}+\tilde{c}_{2}=b(S) .
$$

As a result, (A.18) becomes

$$
\begin{aligned}
T= & C_{2} \rho+b(S) \rho+a_{0 i} \rho u^{i}+a_{1 i} \rho\left(t u^{i}-x^{i}\right)+C_{1 i j} \rho u^{i} x^{j}+b_{0}\left(\frac{1}{2} \rho u^{i} u_{i}+\frac{1}{2} n \kappa(S) \rho^{1+2 / n}\right) \\
& +b_{1}\left(t\left(\frac{1}{2} \rho u^{i} u_{i}+\frac{1}{2} n \kappa(S) \rho^{1+2 / n}\right)-\frac{1}{2} \rho x^{i} u_{i}\right)+b_{2}\left(t^{2}\left(\frac{1}{2} \rho u^{i} u_{i}+\frac{1}{2} n \kappa(S) \rho^{1+2 / n}\right)-t \rho x^{i} u_{i}\right)
\end{aligned}
$$

with arbitrary constants $a_{0 i}, a_{1 i}, b_{0}, b_{1}, b_{2}, C_{1 i j}=-C_{1 j i}, C_{2}$, and an arbitrary function $b(S)$. Also, (A.38) and (A.39) respectively reduce to

$$
P(\rho, S)=\kappa(S) \rho^{1+2 / n}, \quad e(\rho, S)=\frac{1}{2} n \kappa(S) \rho^{2 / n} .
$$

\section{Appendix B. Proof of Theorem 3.1 and Propositions 4.2 And 4.3}

To begin, we write out the component form of the fluid curl, the vorticity scalar and vector, along with their transport equations:

$$
\begin{aligned}
& \boldsymbol{\leftrightarrow} \leftrightarrow \omega^{i j}=\frac{1}{2}\left(u^{i j}, u^{j}{ }^{i}\right)=u^{[i j]}, \\
& \varpi \leftrightarrow \varpi=\epsilon^{i_{1} j_{1} \cdots i_{m} j_{m}} \omega_{i_{1} j_{1}} \cdots \omega_{i_{m} j_{m}}, \quad m=n / 2, \\
& \varpi \leftrightarrow \varpi^{i}=\epsilon^{i j_{1} k_{1} \cdots j_{m} k_{m}} \omega_{j_{1} k_{1}} \cdots \omega_{j_{m} k_{m}}, \quad m=(n-1) / 2, \\
& \omega_{t}^{i j}=\left(u_{k} \omega^{k i}\right)_{,}^{j}-\left(u_{k} \omega^{k j}\right)_{,}^{i}=u_{k}{ }^{i} \omega^{j k}-u_{k}{ }^{j} \omega^{i k}-u^{k} \omega^{i j}, k \\
& \varpi_{t}=-\left(\varpi u^{i}+\rho^{-1} w^{i j} P_{, j}\right)_{, i}=-\varpi u_{, i}^{i}-u^{i} \varpi_{, i}+\rho^{-2} P_{S} w^{j k} \rho_{, j} S_{, k}, \\
& \varpi_{t}^{i}=\left(\varpi^{j} u^{i}-\varpi^{i} u^{j}-\rho^{-1} W^{i j k} P_{, k}\right)_{, j}=\varpi^{j} u_{, j}^{i}-\varpi^{i} u^{j}{ }_{, j}-u^{j} \varpi_{, j}^{i}+\rho^{-2} P_{S} W^{i j k} \rho_{, j} S_{, k} .
\end{aligned}
$$


In addition, we will need the component form for

$$
\begin{aligned}
& \mathbf{w} \leftrightarrow w^{i j}=\epsilon^{i j i_{1} j_{1} \cdots i_{m-1} j_{m-1}} \omega_{i_{1} j_{1}} \cdots \omega_{i_{m-1} j_{m-1}}, \quad m=n / 2, \\
& \mathbf{W} \leftrightarrow W^{i j k}=\epsilon^{i j k j_{1} k_{1} \cdots j_{m-1} k_{m-1}} \omega_{j_{1} k_{1}} \cdots \omega_{j_{m-1} k_{m-1}}, \quad m=(n-1) / 2, \\
& \nabla \varpi \leftrightarrow \varpi_{, i}=w^{j k} \omega_{j k, i}, \quad \nabla \varpi \leftrightarrow \varpi^{j},{ }_{i}=W^{j k l} \omega_{k l, i},
\end{aligned}
$$

along with the identities

$$
\begin{aligned}
& w^{(i j)}=0, \quad w^{i j}{ }_{, j}=0, \quad n w^{i j} \omega_{k j}=\varpi \delta_{k}^{i} \\
& W^{i(j k)}=W^{(i j) k}=0, \quad W^{i j k}{ }_{, k}=0, \\
& \varpi^{i}{ }_{, i}=0 .
\end{aligned}
$$

Here $\epsilon^{i_{1} \cdots i_{n}}=\epsilon^{\left[i_{1} \cdots i_{n}\right]} \leftrightarrow \boldsymbol{\epsilon}, \delta_{i j}=\delta_{(i j)} \leftrightarrow \mathbf{g}$ are the Cartesian components of the spatial orientation tensor and the Euclidean metric tensor on $\mathbb{R}^{n}$; round brackets denote symmetrization of the enclosed indices, and square brackets denote antisymmetrization.

We proceed by explicitly solving the determining equations (2.10) for conserved densities of vorticity type in even and odd dimensions $n>1$.

Case $n=2 m$ : For a conserved density of the form $T\left(S, \rho, u^{i}, \varpi\right)$, the time derivative is given by

$$
\mathcal{D}_{t} T=D_{i}\left(-u^{i} T\right)+u_{, i}^{i} A-\rho^{-1}\left(P_{\rho} \rho_{,}^{i}+P_{S} S_{,}^{i}\right) T_{u^{i}}+\rho^{-2} P_{S} w^{i j} \rho_{, i} S_{, j} T_{\varpi}
$$

after use of identities (B.10), where

$$
A=T-\rho T_{\rho}-\varpi T_{\varpi}
$$

We begin by substituting (B.13) into the determining equations

$$
\begin{aligned}
0=E_{S}\left(\mathcal{D}_{t} T\right)= & u^{i j} \rho^{-2} P_{S}\left(\rho T_{u^{i} u^{j}}+m \rho,{ }^{k} w_{i k} T_{u^{j} \varpi}\right)+\rho,{ }^{i} \rho^{-2} P_{S}\left(\rho T_{u^{i} \rho}+\frac{1}{2} \varpi T_{u^{i} \varpi}-T_{u^{i}}-f T_{u^{i} S}\right) \\
& +\varpi,{ }^{i} \rho^{-2} P_{S}\left(\rho T_{u^{i} \varpi}-m w_{i j} \rho^{j}{ }^{j} T_{\varpi \varpi}\right)+\operatorname{tr} u A_{S}
\end{aligned}
$$

and

$$
\begin{aligned}
0=E_{\rho}\left(\mathcal{D}_{t} T\right)= & u^{i j} \rho^{-2} P_{S}\left(f T_{u^{i} u^{j}}-m S,{ }^{k} w_{i k} T_{u^{j} \varpi}\right)+S_{,}{ }^{i} \rho^{-2} P_{S}\left(T_{u^{i}}-\frac{1}{2} \varpi T_{u^{i} \varpi}-\rho T_{u^{i} \rho}\right) \\
& +\varpi,{ }^{i} \rho^{-2} P_{S}\left(f T_{u^{i} \varpi}-m w_{i j} S,{ }^{j} T_{\varpi \varpi}\right)+\operatorname{tr} u A_{\rho}
\end{aligned}
$$

where we have introduced the notation

$$
\operatorname{tr} u=\delta_{i j} u^{i j}=u_{, i}^{i}, \quad u^{i j}=\frac{1}{2}\left(u^{i j},{ }^{j}+u^{j}{ }_{,}^{i}\right)=u^{(i j)}, \quad f=\rho P_{\rho} / P_{S} .
$$

Since $T$ does not contain any derivatives of $\varpi, S$ and $\rho$, the coefficients of the separate terms $\varpi^{i}{ }^{i},{ }^{j}, \varpi_{,}^{i} \rho{ }^{j}$, and $\varpi,{ }^{i}$ in both (B.15) and (B.16) must vanish, yielding

$$
T_{\varpi \varpi}=0, \quad T_{u^{i} \varpi}=0 .
$$

Integrating ( $(\overline{\mathrm{B} .18})$, and dropping a kinematic term $c\left(S, \rho, u^{i}\right)$ that does not involve $\varpi$, we get

$$
T=a(S, \rho) \varpi .
$$

Then $(\mathrm{B} .15)$ and $(\mathrm{B} .16)$ reduce to $0=\operatorname{tr} u A_{S}=\operatorname{tr} u A_{\rho}$, and hence we have $0=A_{S}=A_{\rho}$ which gives

$$
\begin{aligned}
\left(\rho a_{\rho}\right)_{S}= \\
\left(\rho a_{\rho}\right)_{\rho}=0
\end{aligned}
$$


Integration of these first-order linear equations for $a_{\rho}$ yields

$$
a=b(S)+c \ln \rho, \quad c=\text { const.. }
$$

Thus from (B.19) and (B.21) we have

$$
T=b(S) \varpi+c \varpi \ln \rho
$$

and

$$
\mathcal{D}_{t} T=D_{i}\left(-u^{i} T\right)-c \varpi u_{, i}^{i}+(b(S)+c \ln \rho) \rho^{-2} P_{S} w^{i j} \rho_{, i} S_{, j} .
$$

The third term in (B.23) can be written as a spatial divergence $-D_{i}\left(B(S, \rho) w^{i j} \rho_{, j}\right)=$ $w^{i j} B_{S} \rho_{, i} S_{, j}$ through the identities (B.10), where $B(S, \rho)$ satisfies $B_{S}=\rho^{-2}(b(S)+c \ln \rho) P_{S}$. Thus, (B.23) becomes

$$
\mathcal{D}_{t} T=-D_{i}\left(u^{i} T+B w^{i j} \rho_{, j}\right)-c \varpi u_{, i}^{i} .
$$

Now we substitute (B.24) into the final determining equation

$$
0=E_{u^{i}}\left(\mathcal{D}_{t} T\right)=c \varpi_{, i}+\operatorname{mctr} u_{,}^{j} w_{j i}
$$

Since $\varpi$ has no dependence on $u^{i j}$ (which is linearly independent of $\omega^{i j}$ ), we obtain

$$
c=0 \text {. }
$$

Therefore, (B.26) and (B.22) give the result

$$
T=b(S) \varpi
$$

with

$$
\mathcal{D}_{t} T=-D_{i}\left(u^{i} b(S) \varpi+B(S, \rho) w^{i j} \rho_{, j}\right), \quad B(S, \rho)=\rho^{-2} \int b(S) P_{S}(S, \rho) d S .
$$

We now note that if $b=$ const. then (B.27) is a trivial conserved density

$$
T=b \varpi=b\left(w^{i j} u_{j}\right)_{i}=D_{i}\left(b w^{i j} u_{j}\right) .
$$

Its corresponding moving-flux $\xi^{i}=b \rho^{-2} P w^{i j} \rho_{, j}$ is non-vanishing due to the form of (B.28).

This completes the proof in the even-dimensional case.

Case $n=2 m+1$ : For a conserved density $T\left(S, \rho, u^{i}, \varpi^{i}\right)$, similarly to the previous case, its time derivative is given by

$$
\mathcal{D}_{t} T=D_{i}\left(-u^{i} T\right)+u_{, i}^{i}\left(T-\rho T_{\rho}-\varpi^{j} T_{\varpi^{j}}\right)-\rho^{-1}\left(P_{\rho} \rho^{i}{ }^{i}+P_{S} S_{,}^{i}\right) T_{\rho u^{i}}+\rho^{-2} P_{S} W^{i j k} \rho_{, j} S_{, k} T_{\varpi^{i}} .
$$

The determining equations $0=E_{S}\left(\mathcal{D}_{t} T\right)$ and $0=E_{\rho}\left(\mathcal{D}_{t} T\right)$ then yield

$$
\begin{aligned}
0= & \varpi^{i j}, \rho^{-2} P_{S}\left(\rho T_{u^{j} \varpi^{i}}+\rho_{,}^{l} W_{i l}{ }^{k} T_{\varpi^{k} \varpi^{j}}\right)+\rho^{-2} P_{S} \rho^{i}{ }^{i}\left(\rho T_{u^{i} \rho}-T_{u^{i}}-f T_{u^{i} S}-W_{i j}{ }^{k} \omega^{j l} T_{u^{l} \varpi^{k}}\right) \\
& +u^{i j}\left(\varpi_{j} T_{\varpi^{i} S}+\rho^{-1} P_{S} \rho T_{u^{i} u^{j}}+\rho^{-2} P_{S} \rho,{ }^{l} W_{i l}{ }^{k} T_{\varpi^{k} u^{j}}\right)+\operatorname{tr} u\left(T_{S}-\rho T_{\rho S}-\varpi^{i} T_{\varpi^{i} S}\right)
\end{aligned}
$$

and

$$
\begin{aligned}
0= & \varpi^{i j}, \rho^{-2} P_{S}\left(f T_{u^{j} \varpi^{i}}-S_{,}^{l} W_{i l}^{k} T_{\varpi^{k} \varpi^{j}}\right)+\rho^{-2} P_{S} S^{i},\left(f T_{u^{i} S}+T_{u^{i}}-\rho T_{u^{i} \rho}+W_{i j}{ }^{k} \omega^{j l} T_{u^{l} \varpi^{k}}\right) \\
& +u^{i j}\left(\varpi_{j} T_{\varpi^{i} \rho}+\rho^{-1} P_{\rho} T_{u^{i} u^{j}}-\rho^{-2} P_{S} S^{l}, W_{i l}{ }^{k} T_{\varpi^{k} u^{j}}\right)-\operatorname{tr} u\left(\rho T_{\rho \rho}+\varpi^{i} T_{\rho \varpi^{i}}\right)
\end{aligned}
$$

using the notation (B.17). Since $T$ does not contain any derivatives of $\varpi^{i}$, the first term in both (B.31) and (B.32) must vanish modulo the identity (B.12). This implies

$$
\begin{aligned}
& T_{\varpi^{j} \varpi^{k}}=0, \\
& T_{u^{i} \varpi^{j}}=a\left(\rho, u^{k}, \varpi^{k}\right) \delta_{i j} .
\end{aligned}
$$


Applying the derivative operator $\partial_{u^{k}}$ to $(\overline{\mathrm{B} .34})$, antisymmetrizing in $[j k]$, and taking the trace over $(i j)$, we get $(n-1) a_{u^{k}}=0$. Hence, in $n>1$ dimensions,

$$
a_{u^{k}}=0 .
$$

By also applying the derivative operator $\partial_{\varpi^{k}}$ to (B.34), we similarly get

$$
a_{\varpi^{k}}=0 \text {. }
$$

Integration of (B.33), (B.34), (B.35), (B.36) then yields

$$
T=a(S, \rho) u^{i} \varpi_{i}+b_{i}(S, \rho) \varpi^{i} .
$$

Here we have dropped an integration constant $c\left(S, \rho, u^{i}\right)$ since it does not involve $\varpi^{i}$ (i.e. it is of kinematic form).

The determining equations (B.31) and (B.32) thereby reduce to

$$
\begin{aligned}
& 0=u^{i j} B_{i S} \varpi_{j}-\rho \operatorname{tr} u \varpi^{i} B_{i S \rho}+\rho_{,}{ }^{i} \varpi_{i} \rho^{-2} A, \\
& 0=u^{i j} B_{i \rho} \varpi_{j}-\operatorname{tr} u \varpi^{i}\left(\rho B_{i \rho \rho}+B_{i \rho}\right)-S,{ }^{i} \varpi_{i} \rho^{-2} A,
\end{aligned}
$$

with coefficients

$$
\begin{aligned}
& A=\left(\rho a_{\rho}-f a_{S}-(m+1) a\right) P_{S}, \\
& B_{i}=a u_{i}+b_{i} .
\end{aligned}
$$

In (B.38) and (B.39) the respective coefficients of the terms $\rho,{ }^{i}$ and $S,{ }^{i}$ must vanish, which yields $A=0$. Since $P_{S} \not \equiv 0$, we get

$$
0=\rho a_{\rho}-f a_{S}-(m+1) a .
$$

Then, since the coefficient of $u^{i j}$ must vanish in both (B.38) and (B.39), we obtain

$$
\begin{aligned}
& \varpi_{(j} B_{i) S}=\varpi^{k} \rho B_{k \rho S} \delta_{i j}, \\
& \varpi_{(j} B_{i) \rho}=\varpi^{k}\left(\rho B_{k \rho}\right)_{\rho} \delta_{i j} .
\end{aligned}
$$

By taking the product of $\left(\right.$ B.43) with $\varpi_{l} \varpi_{h}$ and antisymmetrizing in $[i h]$ and $[j l]$, we find $\rho \varpi^{k} B_{k \rho S} \varpi_{[h} \delta_{i] j[} \varpi_{l]}=0$. This implies $B_{k \rho S}=0$. The same antisymmetric product applied to $(\underline{B .44})$ then implies $\left(\rho B_{k \rho}\right)_{\rho}=0$. Hence $(\underline{\mathrm{B} .43})$ and $(\underline{\mathrm{B} .42})$ become $\varpi_{(j} B_{i) S}=\varpi_{(j} B_{i) \rho}=0$, which yields

$$
B_{i S}=B_{i \rho}=0 .
$$

From (B.41) and (B.42) we thus obtain

$$
a=0, \quad b^{i}=\text { const.. }
$$

Hence (B.37) reduces to

$$
T=b_{i} \varpi^{i}=D_{j}\left(b_{i} W^{i j k} u_{k}\right)
$$

which is a trivial conserved density, with

$$
\mathcal{D}_{t} T=D_{i}\left(b_{j}\left(2 u^{[j} \varpi^{i]}-W^{i j k}\left(\rho^{-1}\right)_{, k}\right)\right)
$$

as given by (B.30) combined with the identities $u^{i}{ }_{j} \varpi^{j}=D_{j}\left(u^{i} \varpi^{j}\right)$ and $\rho^{-2} P_{S} W^{i j k} \rho_{, j} S_{, k}=$ $-W^{i j k}\left(\rho^{-1}\right)_{, j} P_{, k}=D_{k}\left(-W^{i j k} P\left(\rho^{-1}\right)_{, j}\right)$ obtained via (B.12) and (B.11). The corresponding moving-flux has the form $\xi^{i}=-b_{j}\left(u^{j} \varpi^{i}+\rho^{-2} W^{i j k} \rho_{, k}\right)$ which is non-vanishing.

This completes the proof in the odd-dimensional case. 


\section{REFERENCES}

[1] Anco, S.C., Conservation laws of scaling-invariant field equations, J. Phys. A: Math. Gen., 36 (2003), 8623-8638.

[2] Anco, S.C. and Bluman, G., Direct construction of conservation laws from field equations, Phys. Rev. Lett. 78 (1997), 2869-2873.

[3] Anco, S.C. and Bluman, G., Direct construction method for conservation laws of partial differential equations. Part I: Examples of conservation law classifications, Eur. J. Appl. Math. 13 (2002), 545-66.

[4] Anco, S.C. and Bluman, G., Direct construction method for conservation laws of partial differential equations. Part II: General treatment, Eur. J. Appl. Math. 13 (2002), 567-85.

[5] Anco, S.C. and Dar, A., Classification of conservation laws of compressible isentropic fluid flow in $n>1$ spatial dimensions, Proc. Roy. Soc. A 464 (2009), 2461-2488.

[6] Anco, S.C. and Dar, A., Lower-degree conservation laws in fluid flow. In preparation.

[7] Arnold, V.I., Sur la géométrie différentielle des groupes de Lie de dimension infinie et ses applications à l'hydrodynamique des fluides parfaits, Ann. Inst. Fourier 16 (1966), 316-361.

[8] Arnold, V.I., The Hamiltonian nature of the Euler equation in the dynamics of rigid body and of an ideal fluid, Uspekhi Mat. Nauk 24 (1969), No. 3, 225-226.

[9] Arnold, V.I. and Khesin, B.A., Topological Methods in Hydrodynamics, Springer-Verlag, 1998.

[10] Bluman, G., Cheviakov, A., Anco, S.C., Applications of Symmetry Methods to Partial Differential Equations, Springer, 2009.

[11] Bluman, G., Temerchaolu, Anco, S.C., New conservations laws obtained directly from symmetry action on a known conservation law, J. Math. Anal. Appl. 322 (2006), 233-250.

[12] Dezin, A.A., Invariant forms and some structure properties of the Euler equations of hydrodynamics, Zeit. Anal. Anwend. (in Russian) 2 (1983), 401-409.

[13] Ibragimov, N.H., Conservation laws in hydrodynamics, Dokl. Akad. Nauk USSR, 210, No. 6: 1307-1309, 1973. English transl., Soviet Physics Dokl., 18 (1973-1974).

[14] Ibragimov, N.H., CRC Handbook of Lie Group Analysis of Differential Equations Vol. 1,2,3, CRC Press, 1994-1996.

[15] Khesin, B.A. and Chekanov Y.V., Invariants of the Euler equations for ideal or barotropic hydrodynamics and superconductivity in D dimensions, Physica D 40 (1989), 119-131.

[16] Kupershmidt, B.A., The Variational Principles of Dynamics, Advanced Series in Mathematical Physics vol. 13, World Scientific, 1992.

[17] Landau, L.D. and Lifshitz, E.M., Fluid Mechanics, Pergamon, 1968.

[18] Lighthill, M.J., An informal introduction to theoretical fluid mechanics, Oxford University Press, 1986.

[19] Olver, P.J., Applications of Lie Groups to Differential Equations, Springer-Verlag, 1993.

[20] Olver, P.J. and Nutku, Y.J., Hamiltonian structures for systems of hyperbolic conservation laws, J. Math. Phys. 29 (1988), 1610-1619.

[21] Ovsyannikov, L.V., Group properties of differential equations (Russian), USSR Academy of Sciences, Novosibirsk, 1962.

[22] Serre, D., Invariants et dégénérescenc symplectique de l'équation d'Euler des fluids parfaits incompressibles, C.R. Acad. Sci. Paris, Sér. A 298 (1984), 349.

[23] Verosky, J., Higher-order symmetries of the compressible one-dimensional isentropic fluid equations, J. Math. Phys. 25 (1984), 884-888.

[24] Verosky, J., First-order conserved densities for gas dynamics, J. Math. Phys. 27 (1986), 3061-3063.

[25] Verosky, J., The Hamiltonian structure of generalized fluid equations, Lett. Math. Phys. 9 (1985), 51-53.

[26] Whitham, G.B., Linear and nonlinear waves, Wiley, 1974.

E-mail address: sanco@brocku.ca

E-mail address: amanullahdar@hotmail.com 